\title{
WEIGHTED LOCAL MORREY SPACES
}

\author{
Shohei Nakamura, Yoshihiro Sawano and Hitoshi Tanaka
}

Tokyo Metropolitan University, Department of Mathematics and Information Sciences

Minami-Ohsawa 1-1, Hachioji-shi, Tokyo 192-0397, Japan; nakamura-shouhei@ed.tmu.ac.jp

Tokyo Metropolitan University, Department of Mathematics and Information Sciences

Minami-Ohsawa 1-1, Hachioji-shi, Tokyo 192-0397, Japan; ysawano@tmu.ac.jp and Department of Mathematical Analysis and Theory of Functions

Peoples Friendship University of Russia (RUDN University)

6 Miklukho-Maklay St, Moscow, 117198 Russia

National University Corporation Tsukuba University of Technology

Research and Support Center on Higher Education for the Hearing and Visually Impaired

Kasuga 4-12-7, Tsukuba 305-8521, Japan; htanaka@k.tsukuba-tech.ac.jp

\begin{abstract}
We discuss the boundedness of linear and sublinear operators in two types of weighted local Morrey spaces. One is defined by Natasha Samko in 2008. The other is defined by Yasuo Komori-Furuya and Satoru Shirai in 2009. We characterize the class of weights for which the Hardy-Littlewood maximal operator is bounded. Under a certain integral condition it turns out that the singular integral operators are bounded if and only if the Hardy-Littlewood maximal operator is bounded. As an application of the characterization, the power weight function $|\cdot|^{\alpha}$ is considered. The condition on $\alpha$ for which the Hardy-Littlewood maximal operator is bounded can be described completely.
\end{abstract}

\section{Introduction}

The aim of this paper is to characterize the class of weights for which the HardyLittlewood maximal operator $M$ is bounded on the weighted local Morrey space $L \mathcal{M}_{q}^{p}(1, w)$ of Samko type and on the weighted local Morrey space $L \mathcal{M}_{q}^{p}(w, w)$ of Komori-Shirai type. A similar characterization is obtained for the singular integral operators, the fractional integral operators and the fractional maximal operators. Here and below by a weight we mean a locally integrable function on $\mathbf{R}^{n}$ which is almost everywhere positive.

We shall consider all cubes in $\mathbf{R}^{n}$ which have their sides parallel to the coordinate axes. We denote by $\mathcal{Q}$ the family of all such cubes. For a cube $Q \in \mathcal{Q}$ we use $\ell(Q)$ to denote the sides length of $Q, c(Q)$ to denote the center of $Q,|Q|$ to denote the volume of $Q$ and $c Q$ to denote the cube with the same center as $Q$ but with side-length $c \ell(Q)$.

The class $A_{p}$ with $1<p<\infty$ is defined to the set of all weights $w$ for which

$$
\sup _{Q \in \mathcal{Q}} \frac{1}{|Q|} \int_{Q} w(x) \mathrm{d} x\left(\int_{Q} w(x)^{-\frac{1}{p-1}} \mathrm{~d} x\right)^{p-1}<\infty .
$$

This class $A_{p}$, initiated by Muckenhoupt [42], characterizes the condition for which there exists a constant $C>0$ such that

$$
\int_{\mathbf{R}^{n}} M f(x)^{p} w(x) \mathrm{d} x \leq C \int_{\mathbf{R}^{n}}|f(x)|^{p} w(x) \mathrm{d} x
$$

https://doi.org/10.5186/aasfm.2020.4504

2010 Mathematics Subject Classification: Primary 42B25, 42B35, 26A33.

Key words: Local Morrey spaces of Samko type, local Morrey spaces of Komori-Shirai type, weights. 
for all measurable functions $f$, where $M$ is the Hardy-Littlewood maximal operator defined by

$$
M f(x) \equiv \sup _{Q \in \mathcal{Q}} \frac{\chi_{Q}(x)}{|Q|} \int_{Q}|f(y)| \mathrm{d} y, \quad x \in \mathbf{R}^{n} .
$$

The Hardy-Littlewood maximal operator $M$ plays a fundamental role in harmonic analysis. The Riesz transform, which is given by

$$
R_{j} f(x) \equiv \lim _{\varepsilon \downarrow 0} \int_{\mathbf{R}^{n} \backslash B(x, \varepsilon)} \frac{x_{j}-y_{j}}{|x-y|^{n+1}} f(y) \mathrm{d} y,
$$

is also important in harmonic analysis. Importantly, if $w \in A_{p}$, then

$$
\int_{\mathbf{R}^{n}}\left|R_{j} f(x)\right|^{p} w(x) \mathrm{d} x \leq C \int_{\mathbf{R}^{n}}|f(x)|^{p} w(x) \mathrm{d} x
$$

for all $f \in L_{\mathrm{c}}^{\infty}$.

Recently, more and more Banach lattices come into play in harmonic analysis. For example, local Morrey spaces play important role to describe the real interpolation of weighted Lebesgue spaces, which covers the off-range of the Stein-Weiss interpolation theorem [3].

Let $1 \leq q \leq p<\infty$. The local Morrey norm $\|\cdot\|_{L \mathcal{M}_{q}^{p}}$ is given by

$$
\|f\|_{L \mathcal{M}_{q}^{p}} \equiv \sup _{R>0}\left|[-R, R]^{n}\right|^{\frac{1}{p}-\frac{1}{q}}\left\|f \chi_{[-R, R]^{n}}\right\|_{L^{q}}
$$

for a measurable function $f$. The local Morrey space $L \mathcal{M}_{q}^{p}$ is the set of all the measurable functions $f$ for which the norm $\|f\|_{L \mathcal{M}_{q}^{p}}$ is finite. Following the notation in the works $[4,10,49]$, we define weighted local Morrey spaces as follows: For a measurable function $f$ and the weights $u$ and $w$, we write

$$
\|f\|_{L \mathcal{M}_{q}^{p}(u, w)} \equiv \sup _{R>0} u\left([-R, R]^{n}\right)^{\frac{1}{p}-\frac{1}{q}}\left\|f \chi_{[-R, R]^{n}}\right\|_{L^{q}(w)} .
$$

The two-weight local Morrey space $\operatorname{LM}_{q}^{p}(u, w)$ is the set of all measurable functions $f$ for which the norm $\|f\|_{L \mathcal{M}_{q}^{p}(u, w)}$ is finite. If $u=1$, then we call $L \mathcal{M}_{q}^{p}(1, w)$ the local Morrey space of Samko type based on [47, 48] and if $u=w$, then we call $\operatorname{LM}_{q}^{p}(w, w)$ the local Morrey space of Komori-Shirai type based on [37]. When $p=q$, $L \mathcal{M}_{p}^{p}(u, w)=L^{p}(w)$ and hence $\operatorname{LM}_{p}^{p}(1, w)=L \mathcal{M}_{p}^{p}(w, w)=L^{p}(w)$ with coincidence of norms. So, in this case the theory of $A_{p}$ applies readily.

The weighted local Morrey space $\operatorname{LM}_{q}^{p}(u, w)$ is a contrast of the weighted Morrey space $\mathcal{M}_{q}^{p}(u, w)$ which consists of all measurable functions $f$ for which the norm

$$
\|f\|_{\mathcal{M}_{q}^{p}(u, w)} \equiv \sup _{Q \in \mathcal{Q}} u(Q)^{\frac{1}{p}-\frac{1}{q}}\left\|f \chi_{Q}\right\|_{L^{p}(w)}
$$

is finite. If $u(x)=|x|^{\beta}$ and $w(x)=1$ or $u(x)=v(x)=|x|^{\beta}$ with $\beta \in \mathbf{R}$, then we say that $L_{\mathcal{M}}^{p}(u, w)$ is the power weighted local Morrey space and that $\mathcal{M}_{q}^{p}(u, w)$ is the power weighted Morrey space.

In this paper, assuming that $1<q<p<\infty$, we seek a characterization for the Hardy-Littlewood maximal operator $M$ to be bounded mainly on $L \mathcal{M}_{q}^{p}(1, w)$ and $\operatorname{LM}_{q}^{p}(w, w)$, motivated by the characterization due to Muckenhoupt.

For $\nu \in \mathbf{Z}$ and $m=\left(m_{1}, m_{2}, \ldots, m_{n}\right) \in \mathbf{Z}^{n}$, we define $Q_{\nu m} \equiv \prod_{j=1}^{n}\left[\frac{m_{j}}{2^{\nu}}, \frac{m_{j}+1}{2^{\nu}}\right)$. Denote by $\mathcal{D}=\mathcal{D}\left(\mathbf{R}^{n}\right)$ the set of such cubes. The elements in $\mathcal{D}$ are called dyadic cubes. Denote by dist $_{\infty}$ the $\ell^{\infty}$-distance on $\mathbf{R}^{n}$. We define the base $L \mathcal{Q}$ of cubes by

$$
L \mathcal{Q} \equiv\left\{Q \in \mathcal{D}: \operatorname{dist}_{\infty}(\{0\}, Q)=\ell(Q)\right\} .
$$


We notice that the dyadic cubes in $L \mathcal{Q}$ are pairwise disjoint and that

$$
\bigcup\{Q: Q \in L \mathcal{Q}\}=\mathbf{R}^{n} \backslash\{0\}
$$

We also notice that the number of dyadic cubes in $L \mathcal{Q}$ with the same side length is $2^{n}\left(2^{n}-1\right)$.

We define the weight class $\mathcal{G}$ by the set of all weights $u$ that satisfy the following:

(i) $u$ is doubling and reverse doubling at the origin, that is, there exist $\beta>\alpha>1$ such that

$$
\alpha u\left([-R, R]^{n}\right) \leq u\left([-2 R, 2 R]^{n}\right) \leq \beta u\left([-R, R]^{n}\right), \quad R>0 ;
$$

(ii) $u$ is doubling with respect to $L \mathcal{Q}$ in the following sense: there exists $\gamma>1$ such that

for all $Q \in L \mathcal{Q}$.

$$
u\left([-2 \ell(Q), 2 \ell(Q)]^{n}\right) \leq \gamma u(Q)
$$

Our results are besed upon the following structure of weighted local Morrey spaces.

Lemma 1.1. Let $1<q<p<\infty$ and let $u$ and $w$ be weights. Assume that $u \in \mathcal{G}$. Then

$$
\|f\|_{L \mathcal{M}_{q}^{p}(u, w)} \sim \sup _{Q \in L \mathcal{Q}} u(Q)^{\frac{1}{p}-\frac{1}{q}}\left\|f \chi_{Q}\right\|_{L^{q}(w)}
$$

holds for any measurable function $f$.

Lemma 1.1 will be proved in Section 2.

Let $1<q<p<\infty$, and let $u$ and $w$ be weights. For a measurable function $g$ its $L \mathcal{M}_{q}^{p}(u, w)$-associate norm $\|g\|_{L \mathcal{M}_{q}^{p}(u, w)^{\prime}}$ is defined by

$$
\|g\|_{L \mathcal{M}_{q}^{p}(u, w)^{\prime}} \equiv \sup \left\{\|f \cdot g\|_{L^{1}}: f \in L \mathcal{M}_{q}^{p}(u, w),\|f\|_{L \mathcal{M}_{q}^{p}(u, w)} \leq 1\right\} .
$$

The space $L \mathcal{M}_{q}^{p}(u, w)^{\prime}$ collects all measurable functions $g$ for which the norm $\|g\|_{L \mathcal{M}_{q}^{p}(u, w)^{\prime}}$ is finite. The space $\operatorname{LM}_{q}^{p}(u, w)^{\prime}$ is called the Köthe dual of $\operatorname{LM}_{q}^{p}(u, w)$ or the associated space of $L \mathcal{M}_{q}^{p}(u, w)$. Below, we list our results.

Theorem 1.2. Let $1<q<p<\infty$ and let $u$ and $w$ be weights. Assume that $u \in \mathcal{G}$. Then the following are equivalent:

(1) The Hardy-Littlewood maximal operator $M$ is bounded on $\operatorname{LM}_{q}^{p}(u, w)$;

(2) there exists a constant $C$ which is independent of $Q \in L \mathcal{Q}$ such that

$$
\sup _{R \in \mathcal{Q}, R \subset 2 Q} \frac{1}{|R|}\left\|\chi_{R}\right\|_{L^{q}(w)}\left\|\chi_{R}\right\|_{L^{q}(w)^{\prime}}+\sup _{R \in \mathcal{Q}, R \supset Q} \frac{1}{|R|}\left\|\chi_{Q}\right\|_{L \mathcal{M}_{q}^{p}(u, w)}\left\|\chi_{R}\right\|_{L \mathcal{M}_{q}^{p}(u, w)^{\prime}} \leq C .
$$

As a special case of Theorem 1.2, we obtain the following characterizations of $L \mathcal{M}_{q}^{p}(1, w)$ and $\operatorname{LM}_{q}^{p}(w, w)$.

Corollary 1.3. Let $1<q<p<\infty$ and let $w$ be a weight. Then the following are equivalent:

(1) The Hardy-Littlewood maximal operator $M$ is bounded on $L \mathcal{M}_{q}^{p}(1, w)$;

(2) there exists a constant $C$ which is independent of $Q \in L \mathcal{Q}$ such that

$$
\sup _{R \in \mathcal{Q}, R \subset 2 Q} \frac{1}{|R|}\left\|\chi_{R}\right\|_{L^{q}(w)}\left\|\chi_{R}\right\|_{L^{q}(w)^{\prime}}+\sup _{R \in \mathcal{Q}, R \supset Q} \frac{1}{|R|}\left\|\chi_{Q}\right\|_{L \mathcal{M}_{q}^{p}(1, w)}\left\|\chi_{R}\right\|_{L \mathcal{M}_{q}^{p}(1, w)^{\prime}} \leq C .
$$

Proposition 1.4. Let $1<q<p<\infty$ and let $w$ be a weight. Then the following are equivalent:

(1) The Hardy-Littlewood maximal operator $M$ is bounded on $\operatorname{LM}_{q}^{p}(w, w)$; 
(2) $w \in \mathcal{G}$ and there exists a constant $C$ which is independent of $Q \in L \mathcal{Q}$ such that

$$
\sup _{R \in \mathcal{Q}, R \subset 2 Q} \frac{1}{|R|}\left\|\chi_{R}\right\|_{L^{q}(w)}\left\|\chi_{R}\right\|_{L^{q}(w)^{\prime}}+\sup _{R \in \mathcal{Q}, R \supset Q} \frac{1}{|R|}\left\|\chi_{Q}\right\|_{L \mathcal{M}_{q}^{p}(w, w)}\left\|\chi_{R}\right\|_{L \mathcal{M}_{q}^{p}(w, w)^{\prime}} \leq C .
$$

Our characterization can be applied to singular integral operators including the Riesz transform. A singular integral operator is an $L^{2}$-bounded linear operator $T$ that comes with a function $K: \mathbf{R}^{n} \times \mathbf{R}^{n} \rightarrow \mathbf{C}$ satisfying the following conditions:

(1) (Size condition); For all $x, y \in \mathbf{R}^{n}$,

$$
|K(x, y)| \lesssim|x-y|^{-n}
$$

(2) (Gradient condition); For all $x, y, z \in \mathbf{R}^{n}$ satisfying $|x-z|>2|y-z|$,

$$
|K(x, z)-K(y, z)| \lesssim|x-y|^{-n-1}|y-z| .
$$

(3) Let $f$ be an $L^{2}$-function. For almost all $x \notin \operatorname{supp}(f)$,

$$
T f(x)=\int_{\mathbf{R}^{n}} K(x, y) f(y) \mathrm{d} y .
$$

The function $K$ is called the integral kernel of $T$.

Here and below in this paper we use the following notation: Let $A, B \geq 0$. Then $A \lesssim B$ and $B \gtrsim A$ mean that there exists a constant $C>0$ such that $A \leq C B$, where $C$ depends only on the parameters of importance. The symbol $A \sim B$ means that $A \lesssim B$ and $B \lesssim A$ happen simultaneously.

For the singular integral operators, we have the following characterization.

Theorem 1.5. Assume that $\left\|\chi_{2^{k+1} Q_{0}}\right\|_{\mathrm{LM}_{q}^{p}(u, w)} \geq \alpha\left\|_{2^{k} Q_{0}}\right\|_{\mathrm{L} \mathcal{M}_{q}^{p}(u, w)}, Q_{0} \in \mathrm{L} \mathcal{Q}$ for some $\alpha>1$ independent on $k \in \mathbf{N}$. Let $T$ be a singular integral operator. Let $1<q<p<\infty$ and let $u$ and $w$ be weights. Assume that $u \in \mathcal{G}$ and that there exists a constant $C$ which is independent of $Q \in L \mathcal{Q}$ such that

$$
\sup _{R \in \mathcal{Q}, R \subset 2 Q} \frac{1}{|R|}\left\|\chi_{R}\right\|_{L^{q}(w)}\left\|\chi_{R}\right\|_{L^{q}(w)^{\prime}}+\sup _{R \in \mathcal{Q}, R \supset Q} \frac{1}{|R|}\left\|\chi_{Q}\right\|_{L \mathcal{M}_{q}^{p}(u, w)}\left\|\chi_{R}\right\|_{L \mathcal{M}_{q}^{p}(u, w)^{\prime}} \leq C .
$$

Then $\|T f\|_{L \mathcal{M}_{q}^{p}(u, w)} \lesssim\|f\|_{L \mathcal{M}_{q}^{p}(u, w)}$ for all $f \in L_{\mathrm{c}}^{\infty}$.

The condition on $u$ and $w$ corresponds to the integral condition considered in [44].

Theorem 1.6. Let $1<q<p<\infty$ and let $w$ be a weight. Assume that $u \in \mathcal{G}$ and that $\left\|R_{j} f\right\|_{L \mathcal{M}_{q}^{p}(u, w)} \lesssim\|f\|_{L \mathcal{M}_{q}^{p}(u, w)}$ for all $f \in L_{\mathrm{c}}^{\infty}$ and for all $j=1,2, \ldots, n$. Then there exists a constant $C$ which is independent of $Q \in L \mathcal{Q}$ such that

$$
\sup _{R \in \mathcal{Q}, R \subset 2 Q} \frac{1}{|R|}\left\|\chi_{R}\right\|_{L^{q}(w)}\left\|\chi_{R}\right\|_{L^{q}(w)^{\prime}}+\sup _{R \in \mathcal{Q}, R \supset Q} \frac{1}{|R|}\left\|\chi_{Q}\right\|_{L \mathcal{M}_{q}^{p}(u, w)}\left\|\chi_{R}\right\|_{L \mathcal{M}_{q}^{p}(u, w)^{\prime}} \leq C .
$$

To investigate weighted Morrey spaces of Samko type, we simply let $u=1$ in Theorems 1.5 and 1.6. Meanwhile, we have investigated the sufficiency of the boundedness of the singular integral operators on weighted Morrey spaces of KomoriShirai type. However the necessity is somewhat non-trivial. So, we formulate it.

Proposition 1.7. Let $1<q<p<\infty$, and let $w$ be a doubling weight. Assume in addition that $\left\|R_{j} f\right\|_{L \mathcal{M}_{q}^{p}(w, w)} \lesssim\|f\|_{L \mathcal{M}_{q}^{p}(w, w)}$ for all $f \in L_{\mathrm{c}}^{\infty}$ and for all $j=1,2, \ldots, n$. Then there exists a constant $C$ which is independent of $Q \in L \mathcal{Q}$ such 
that

$$
\sup _{R \in \mathcal{Q}, R \subset 2 Q} \frac{1}{|R|}\left\|\chi_{R}\right\|_{L^{q}(w)}\left\|\chi_{R}\right\|_{L^{q}(w)^{\prime}}+\sup _{R \in \mathcal{Q}, R \supset Q} \frac{1}{|R|}\left\|\chi_{Q}\right\|_{L \mathcal{M}_{q}^{p}(w, w)}\left\|\chi_{R}\right\|_{L \mathcal{M}_{q}^{p}(w, w)^{\prime}} \leq C .
$$

We apply our results to special cases.

Proposition 1.8. Let $1<q<p<\infty$ and let $w(x)=w_{\beta}(x)=|x|^{\beta}$ with $\beta \in \mathbf{R}$. Then the following are equivalent:

(1) The maximal operator $M$ is bounded on $\operatorname{LM}_{q}^{p}(1, w)$;

(2) $-\frac{q}{p} n \leq \beta<n q\left(1-\frac{1}{p}\right)$.

Proposition 1.9. Let $1<q<p<\infty$ and let $w(x)=w_{\beta}(x)=|x|^{\beta}$ with $\beta \in \mathbf{R}$. Then the following are equivalent:

(1) The maximal operator $M$ is bounded on $\operatorname{LM}_{q}^{p}(w, w)$;

(2) $-n<\beta<n(p-1)$;

(3) $w \in A_{p}$.

The ranges obtained in Propositions 1.8 and 1.9 are the same as that for weighted Morrey spaces of Komori-Shirai type and Samko type, respectively.

Proposition 1.10. [53] Let $1<q<p<\infty$ and let $w(x)=w_{\beta}(x)=|x|^{\beta}$ with $\beta \in \mathbf{R}$. Then the following are equivalent:

(1) The maximal operator $M$ is bounded on $\mathcal{M}_{q}^{p}(1, w)$;

(2) $-n \frac{q}{p} \leq \beta<n q\left(1-\frac{1}{p}\right)$.

One of the ways to investigate the boundedness of the operators acting on Morrey spaces is to combine the translation and the boundedness of the operators acting on corresponding local Morrey spaces. Propositions 1.8 and 1.10 are significant in that Proposition 1.10 can not be obtained by the translation of Proposition 1.8.

In [33] Iida and the first author obtained a complete characterization of the dual inequality of Stein type in weighted Morrey spaces $\mathcal{M}_{q}^{p}(1, w)$ of Samko type. See also [30]. Despite the recent works $[44,46,53]$ a complete characterization of the class for which $M$ is bounded on $\mathcal{M}_{q}^{p}(1, w)$ or $\mathcal{M}_{q}^{p}(w, w)$ is still missing.

Proposition 1.11. Let $1<q<p<\infty$ and let $w(x)=w_{\beta}(x)=|x|^{\beta}$ with $\beta \in \mathbf{R}$. Then the following are equivalent:

(1) The maximal operator $M$ is bounded on $\mathcal{M}_{q}^{p}(w, w)$;

(2) $-n<\beta<n(p-1)$;

(3) $w \in A_{p}$.

We can consider the weighted norm inequalities for other operators. Let $I_{\alpha}$ be the fractional integral operator given by

$$
I_{\alpha} f(x) \equiv \int_{\mathbf{R}^{n}} \frac{f(y)}{|x-y|^{n-\alpha}} \mathrm{d} y, \quad x \in \mathbf{R}^{n},
$$

for a nonnegative measurable function $f$. The boundedness of the fractional integral operator can be characterized in a similar way. Let $1<p<s<\infty$. Recall that the class $A_{p, s}$ of weights is defined to the set of all weights for which there exists a constant $C>0$ satisfying

$$
\left\|I_{\alpha} f \cdot w\right\|_{L^{s}} \leq C\|f \cdot w\|_{L^{p}}
$$

for any nonnegative measurable function $f$. 
To extend this boundedness to Morrey spaces, we can consider two types of boundedness. One is due to Spanne [52] and the other is due to Adams [1]. The following theorem corresponds to the result due to Spanne.

Theorem 1.12. Let $1<q<p<\infty, 1<t<s<\infty$ and $0<\alpha<n$ satisfy

$$
\alpha=\frac{n}{p}-\frac{n}{s}=\frac{n}{q}-\frac{n}{t} .
$$

Let $u$ and $w$ be weights such that $u \in \mathcal{G}$. Consider the following statements:

(1) There exists a constant $C>0$ such that

$$
\left\|I_{\alpha} f\right\|_{L \mathcal{M}_{t}^{s}\left(u, w^{t}\right)} \leq C\|f\|_{L \mathcal{M}_{q}^{p}\left(u, w^{q}\right)}
$$

for any nonnegative measurable function $f$;

(2) there exists a constant $C>0$ such that

$$
\left\|M_{\alpha} f\right\|_{L \mathcal{M}_{t}^{s}\left(u, w^{t}\right)} \leq C\|f\|_{L \mathcal{M}_{q}^{p}\left(u, w^{q}\right)}
$$

for any nonnegative measurable function $f$;

(3) there exists a constant $C$ which is independent of $Q \in L \mathcal{Q}$ such that

$$
\sup _{R \in \mathcal{Q}, R \subset 2 Q} \frac{\ell(R)^{\alpha}}{|R|}\left\|\chi_{R}\right\|_{L^{t}\left(w^{t}\right)}\left\|\chi_{R}\right\|_{L^{q}\left(w^{q}\right)^{\prime}} \leq C
$$

and

$$
\sup _{R \in \mathcal{Q}, R \supset Q} \frac{\ell(R)^{\alpha}}{|R|}\left\|\chi_{Q}\right\|_{L \mathcal{M}_{t}^{s}\left(u, w^{t}\right)}\left\|\chi_{R}\right\|_{L \mathcal{M}_{q}^{p}\left(u, w^{q}\right)^{\prime}} \leq C .
$$

Then;

- (1) implies (2) and (3).

- (2) and (3) are equivalent.

- If there exists $\kappa>1$ such that $2\left\|\chi_{Q}\right\|_{\mathrm{LM}_{t}^{s}\left(u, w^{t}\right)} \leq \kappa\left\|_{\kappa Q}\right\|_{\mathrm{L} \mathcal{M}_{t}^{s}\left(u, w^{t}\right)}$ for all cubes $Q \in \mathrm{L} \mathcal{Q}$, then (2) or (3) implies (1).

In the case of power weighted Morrey spaces of Samko type, we have the following characterization of the boundedness.

Proposition 1.13. Let $1<q<p<\infty$ and $1<t<s<\infty$. Assume that

$$
\frac{1}{s}=\frac{1}{p}-\frac{\alpha}{n}, \quad \frac{1}{t}=\frac{1}{q}-\frac{\alpha}{n} .
$$

Then for the power weight $w(x)=w_{\beta}(x)=|x|^{\beta}$ with $\beta \in \mathbf{R}$ the following are equivalent:

(1) The fractional maximal operator $M_{\alpha}$ is bounded from $L \mathcal{M}_{q}^{p}\left(1, w^{q}\right)$ to $L \mathcal{M}_{t}^{s}(1$, $\left.w^{t}\right)$

(2) $-\frac{n}{s} \leq \beta<\frac{n}{p^{\prime}}$, that is, $\beta=\frac{n}{s}$ or $I_{\alpha}: L^{p}\left(w^{p}\right) \rightarrow L^{s}\left(w^{s}\right)$.

We can replace $M_{\alpha}$ by $I_{\alpha}$ once we exclude the case of $\beta=-\frac{n}{s}$ in (2).

In the case of power weighted Morrey spaces of Komori-Shirai type, we have the following well-known characterization of the boundedness.

Proposition 1.14. Let $0<\alpha<n, \beta \in \mathbf{R}, 1<q<p<\infty$ and $1<t<s<\infty$. Assume that

$$
\frac{1}{s}=\frac{1}{p}-\frac{\alpha}{n}, \quad \frac{1}{t}=\frac{1}{s}-\frac{\alpha}{n}
$$

Then for the power weight $w(x)=w_{\beta}(x)=|x|^{\beta}$ with $\beta \in \mathbf{R}$ the following are equivalent: 
(1) The fractional integral operator $I_{\alpha}$ is bounded from $L \mathcal{M}_{q}^{p}\left(w^{t}, w^{q}\right)$ to $L \mathcal{M}_{t}^{s}\left(w^{t}\right.$, $\left.w^{t}\right)$

(2) $-\frac{n}{t}<\beta<\frac{n s}{p^{\prime} t}$.

We can replace $I_{\alpha}$ by $M_{\alpha}$ once we include the case of $\beta=-\frac{n}{t}$ in (2).

It may be interesting to compare these propositions with the following known results for Lebesgue spaces, the case where $p=q$ and hence $s=t$ in Propositions 1.13 and 1.14 .

Proposition 1.15. Let $1<p<s<\infty, 0<\alpha<n$ and $\beta \in \mathbf{R}$. Assume $\frac{1}{s}=\frac{1}{p}-\frac{\alpha}{n}$. Then for the power weight $w(x)=w_{\beta}(x)=|x|^{\beta}$ the following are equivalent:

(1) There exists a constant $C>0$ such that

$$
\left\|I_{\alpha} f \cdot w_{\beta}\right\|_{L^{s}} \leq C\left\|f \cdot w_{\beta}\right\|_{L^{p}}
$$

for all $f \in L_{\mathrm{c}}^{\infty}$.

(2) $-\frac{n}{s}<\beta<\frac{n}{p^{\prime}}$

We can replace $I_{\alpha}$ by $M_{\alpha}$ in the above.

As for weighted Morrey spaces of Samko type, we have the following conclusion:

Proposition 1.16. [46, Proposition 4.1] Let $1<q<p<\infty, 0<\alpha<n$ and $1<t<s<\infty$. Assume that

$$
\frac{1}{s}=\frac{1}{p}-\frac{\alpha}{n}, \quad \frac{q}{p}=\frac{t}{s}
$$

Then for the power weight $w(x)=w_{\beta}(x)=|x|^{\beta}$ with $\beta \in \mathbf{R}$ the following are equivalent:

(1) The fractional maximal operator $M_{\alpha}$ is bounded from $\mathcal{M}_{q}^{p}\left(1, w^{q}\right)$ to $\mathcal{M}_{t}^{s}\left(1, w^{t}\right)$;

(2) $-\frac{n}{s} \leq \beta<\frac{n}{p^{\prime}}$

Proposition 1.17. [46, Proposition 4.2] Let $1<q<p<\infty, 0<\alpha<n$ and $1<t<s<\infty$. Assume that

$$
\frac{1}{s}=\frac{1}{p}-\frac{\alpha}{n}, \quad \frac{q}{p}=\frac{t}{s}
$$

Then for the power weight $w(x)=w_{\beta}(x)=|x|^{\beta}$ with $\beta \in \mathbf{R}$ the following are equivalent:

(1) The fractional integral operator $I_{\alpha}$ is bounded from $\mathcal{M}_{q}^{p}\left(1, w^{q}\right)$ to $\mathcal{M}_{t}^{s}\left(1, w^{t}\right)$;

(2) $-\frac{n}{s}<\beta<\frac{n}{p^{\prime}}$

Proposition 1.18. Let $1<q<p<\infty, 0<\alpha<n$ and $1<t<s<\infty$. Assume that

$$
\frac{1}{s}=\frac{1}{p}-\frac{\alpha}{n}, \quad \frac{1}{t}=\frac{1}{q}-\frac{\alpha}{n}, \quad \beta>-\frac{n}{t} .
$$

Then for the power weight $w(x)=w_{\beta}(x)=|x|^{\beta}$ with $\beta \in \mathbf{R}$ the following are equivalent:

(1) The fractional integral operator $I_{\alpha}$ is bounded from $\mathcal{M}_{q}^{p}\left(w^{t}, w^{q}\right)$ to $\mathcal{M}_{t}^{s}\left(w^{t}, w^{t}\right)$;

(2) $-\frac{n}{t}<\beta<\frac{n s}{p^{\prime} t}$. 
We can replace $I_{\alpha}$ by $M_{\alpha}$ once we include the case of $\beta=-\frac{n}{t}$ in (2).

We remark that in the case of radial functions, Komori and Sato obtained the following result:

Proposition 1.19. [36] Let $1<p<s<\infty, 1<q \leq t<\infty$ and $0<\alpha<n$ satisfy

Let $\beta$ satisfy

$$
\frac{1}{s}=\frac{1}{p}-\frac{\alpha}{n}, \quad \frac{1}{t}=\frac{1}{q}-\frac{\alpha}{n}
$$

$$
\alpha-\frac{n}{q}+\frac{n}{p}<\beta<\frac{n}{q^{\prime}}
$$

Then for the power weight $w(x)=w_{\beta}(x)=|x|^{\beta}$ with $\beta \in \mathbf{R}$,

$$
\left\|I_{\alpha} f \cdot w_{\beta}\right\|_{L \mathcal{M}_{t}^{s}} \leq C\left\|f \cdot w_{\beta}\right\|_{L \mathcal{M}_{q}^{p}}
$$

for all radial functions $f \in L_{\mathrm{c}}^{\infty}$.

There is a huge amount of literatures dealing with weighted Morrey spaces together with their variants. Many researchers investigated the boundedness properties of the linear operators acting on weighted Morrey spaces. such as sublinear operator $[4,12,15,35]$, singular integral operators [14, 35, 63], commutators [17, 12, 35, 59, 61], pseudo-differential operators [26], the square functions [11], Toeplitz operators [56], the fractional integral operators $[12,28,31,32]$ and fractional integrals associated to operators $[51,54,55]$ including the related commutators. Applications to partial differential equations can be found in $[8,19,50]$. Embedding relations together with the envelope are investigated in [22]. A passage to the metric measure spaces is done in [62]. Ye and Wang used the cube testing to get a characterization of a sufficient condition which guarantees the boundedness of the Hardy-Littlewood maximal operator [60]. See [57, 58] for Campanato spaces of Komori-Shirai type.

As for weighted Morrey spaces of Samko type, the boundedness property of the sharp maximal operator, the maximal operator, the singular integral operators, the fractional operarots including the multilinear setting are investigated in $[18,29,44,45,46]$. we can find its application to singular integral equations in [41]. Liu considered the boundedness of the pseudo-differential operators in the setting of generalized Morrey spaces [39]. There are many attempts of obtaining a necessary and sufficient condition for the weighted norm inequality. See [34] for a characterization of a sufficient condition which guarantees the boundedness of the Hardy-Littlewood maximal operator.

The two-weighted Morrey spaces of the type $\mathcal{M}_{q}^{p}(u, v)$ can be found in $[24,43,49]$ including generalized Morrey spaces [5, 9, 12, 13, 20, 21, 25] and their closed subspaces [2].

The remaining part of this paper is organized as follows: In Section 2 we get a characterization of the local Morrey norm $\|\cdot\|_{L \mathcal{M}_{q}^{p}}$. Based on the observation in Section 3, we prove Theorem 1.2 in Section 4. Theorems 1.5 and 1.6 are proved in Section 5. Finally, as apply our results for the power weight $w(x)=|x|^{\alpha}$ in Section 6.

\section{Preliminaries}

2.1. Structure of weighted local Morrey spaces-the proof of Lemma 1.1. We prove Lemma 1.1.

Proof of Lemma 1.1. The proof consists of two auxiliary equivalences. 
We first claim

$$
\|f\|_{L \mathcal{M}_{q}^{p}(u, w)} \sim \sup _{m \in \mathbf{Z}}\left(u\left(\left[-2^{m+1}, 2^{m+1}\right]^{n}\right)^{\frac{1}{p}-\frac{1}{q}}\left\|f \chi_{\left[-2^{m+1}, 2^{m+1}\right]^{n} \backslash\left[-2^{m}, 2^{m}\right]^{n}}\right\|_{L^{q}(w)} .\right.
$$

It is easy to see that

$$
\|f\|_{L \mathcal{M}_{q}^{p}(u, w)} \geq \sup _{m \in \mathbf{Z}} u\left(\left[-2^{m+1}, 2^{m+1}\right]^{n}\right)^{\frac{1}{p}-\frac{1}{q}}\left\|f \chi_{\left[-2^{m+1}, 2^{m+1}\right]^{n} \backslash\left[-2^{m}, 2^{m}\right]^{n}}\right\|_{L^{q}(w)} .
$$

To obtain the reverse inequality, we fix $R>0$. Then if we set $j=\left[1+\log _{2} R\right]$, we have

$$
u\left([-R, R]^{n}\right)^{\frac{1}{p}-\frac{1}{q}}\left\|f \chi_{[-R, R]^{n}}\right\|_{L^{q}(w)} \lesssim u\left(\left[-2^{j}, 2^{j}\right]^{n}\right)^{\frac{1}{p}-\frac{1}{q}}\left\|f \chi_{\left[-2^{j}, 2^{j}\right]^{n}}\right\|_{L^{q}(w)}
$$

thanks to the doubling property of $u$ at the origin. Since $q<p$ and $u$ is reverse doubling at the origin, we have

$$
u\left(\left[-2^{j}, 2^{j}\right]^{n}\right)^{\frac{1}{p}-\frac{1}{q}} \sum_{l=-\infty}^{j-1} u\left(\left[-2^{l}, 2^{l}\right]^{n}\right)^{-\frac{1}{p}+\frac{1}{q}} \sim 1
$$

and hence

$$
\begin{aligned}
u & \left(\left[-2^{j}, 2^{j}\right]^{n}\right)^{\frac{1}{p}-\frac{1}{q}}\left\|f \chi_{\left[-2^{j}, 2^{j}\right]^{n}}\right\|_{L^{q}(w)} \\
\leq & u\left(\left[-2^{j}, 2^{j}\right]^{n}\right)^{\frac{1}{p}-\frac{1}{q}} \sum_{l=-\infty}^{j-1}\left\|f \chi_{\left[-2^{l+1}, 2^{l+1}\right]^{n} \backslash\left[-2^{l}, 2^{l}\right]^{n}}\right\|_{L^{q}(w)} \\
\leq & u\left(\left[-2^{j}, 2^{j}\right]^{n}\right)^{\frac{1}{p}-\frac{1}{q}} \\
& \times \sum_{l=-\infty}^{j-1} u\left(\left[-2^{l}, 2^{l}\right]^{n}\right)^{-\frac{1}{p}+\frac{1}{q}} \cdot u\left(\left[-2^{l}, 2^{l}\right]^{n}\right)^{\frac{1}{p}-\frac{1}{q}}\left\|f \chi_{\left[-2^{l+1}, 2^{l+1}\right]^{n} \backslash\left[-2^{l}, 2^{l}\right]^{n}}\right\|_{L^{q}(w)} \\
\lesssim & u\left(\left[-2^{j}, 2^{j}\right]^{n}\right)^{\frac{1}{p}-\frac{1}{q}} \\
& \times \sum_{l=-\infty}^{j-1} u\left(\left[-2^{l}, 2^{l}\right]^{n}\right)^{-\frac{1}{p}+\frac{1}{q}} \sup _{m \in \mathbf{Z}} u\left(\left[-2^{m+1}, 2^{m+1}\right]^{n}\right)^{\frac{1}{p}-\frac{1}{q}}\left\|f \chi_{\left[-2^{m+1}, 2^{m+1}\right]^{n} \backslash\left[-2^{m}, 2^{m}\right]^{n}}\right\|_{L^{q}(w)} \\
\lesssim & \sup _{m \in \mathbf{Z}} u\left(\left[-2^{m+1}, 2^{m+1}\right]^{n}\right)^{\frac{1}{p}-\frac{1}{q}}\left\|f \chi_{\left[-2^{m+1}, 2^{m+1}\right]^{n} \backslash\left[-2^{m}, 2^{m}\right]^{n}}\right\|_{L^{q}(w) .}
\end{aligned}
$$

Thus,

$$
\|f\|_{L \mathcal{M}_{q}^{p}(u, w)} \lesssim \sup _{m \in \mathbf{Z}} u\left(\left[-2^{m+1}, 2^{m+1}\right]^{n}\right)^{\frac{1}{p}-\frac{1}{q}}\left\|f \chi_{\left[-2^{m+1}, 2^{m+1}\right]^{n} \backslash\left[-2^{m}, 2^{m}\right]^{n}}\right\|_{L^{q}(w)},
$$

which yields (2.1).

Next, we shall verify that

$$
\begin{aligned}
& \sup _{m \in \mathbf{Z}} u\left(\left[-2^{m+1}, 2^{m+1}\right]^{n}\right)^{\frac{1}{p}-\frac{1}{q}}\left\|f \chi_{\left[-2^{m+1}, 2^{m+1}\right]^{n} \backslash\left[-2^{m}, 2^{m}\right]^{n}}\right\|_{L^{q}(w)} \\
& \sim \sup _{Q \in L \mathcal{Q}} u(Q)^{\frac{1}{p}-\frac{1}{q}}\left\|f \chi_{Q}\right\|_{L^{q}(w)} .
\end{aligned}
$$

A simple geometric observation shows that

$$
\begin{aligned}
& u\left(\left[-2^{m+1}, 2^{m+1}\right]^{n}\right)^{\frac{1}{p}-\frac{1}{q}}\left\|f \chi_{\left[-2^{m+1}, 2^{m+1}\right]^{n} \backslash\left[-2^{m}, 2^{m}\right]^{n}}\right\|_{L^{q}(w)} \\
& \leq 2^{n}\left(2^{n}-1\right) \sup _{Q \in L \mathcal{Q}, \ell(Q)=2^{m}} u(Q)^{\frac{1}{p}-\frac{1}{q}}\left\|f \chi_{Q}\right\|_{L^{q}(w)} .
\end{aligned}
$$


Thanks to the doubling condition of $u$ (with respect to $L \mathcal{Q}$ ), we have reverse inequality

$$
u(Q)^{\frac{1}{p}-\frac{1}{q}}\left\|f \chi_{Q}\right\|_{L^{q}(w)} \lesssim u\left(\left[-2^{m+1}, 2^{m+1}\right]^{n}\right)^{\frac{1}{p}-\frac{1}{q}}\left\|f \chi_{\left[-2^{m+1}, 2^{m+1}\right]^{n} \backslash\left[-2^{m}, 2^{m}\right]^{n}}\right\|_{L^{q}(w)}
$$

for all cubes $Q \in L \mathcal{Q}$ with $\ell(Q)=2^{m}$. Thus, (2.2) is verified and the proof of the lemma is completed.

2.2. The Lerner-Hytönen decomposition. To investigate the boundedness property of the singular integral operators in Theorem 1.5, we need the LernerHytönen decomposition.

A collection $\left\{Q_{j}^{k}\right\}_{k \in \mathbf{N}_{0}, j \in J_{k}}$ of dyadic cubes is said to be sparse, if the union

$$
\Omega_{k} \equiv \bigcup_{j \in J_{k}} Q_{j}^{k}, \quad k=1,2, \ldots
$$

satisfies;

$$
\chi_{\Omega_{k+1}}=\sum_{j \in J_{k+1}} \chi_{Q_{j}^{k+1}} \leq \chi_{\Omega_{k}}=\sum_{j \in J_{k}} \chi_{Q_{j}^{k}} \leq 1
$$

and

$$
2\left|\Omega_{k+1} \cap Q_{j}^{k}\right| \leq\left|Q_{j}^{k}\right| \quad\left(j \in J_{k}\right) .
$$

Let $Q_{0}$ be a cube and $f: Q_{0} \rightarrow \mathbf{R}$ be a measurable function. Choose $m_{f}\left(Q_{0}\right)$ so that

$$
\left|\left\{x \in Q_{0}: f(x)>m_{f}\left(Q_{0}\right)\right\}\right|, \quad\left|\left\{x \in Q_{0}: f(x)<m_{f}\left(Q_{0}\right)\right\}\right| \leq \frac{1}{2}\left|Q_{0}\right| .
$$

Note that $m_{f}\left(Q_{0}\right)$ is not determined uniquely. The quantity $m_{f}\left(Q_{0}\right)$ is called the median of $f$ over $Q_{0}$. The mean oscillation of $f$ over a cube $Q$ of level $\lambda \in(0,1)$ is given by;

$$
\omega_{\lambda}(f ; Q) \equiv \inf _{c \in \mathbf{C}}\left((f-c) \chi_{Q}\right)^{*}(\lambda|Q|)
$$

where $*$ denotes the decreasing rearrangement for functions. Hytönen showed that there exists a sparse family $\left\{Q_{j}^{k}\right\}_{k \in \mathbf{N} \cup\{0\}, j \in J_{k}} \subset \mathcal{D}\left(Q_{0}\right)$ such that

$$
\left|f(x)-m_{f}\left(Q_{0}\right)\right| \leq 2 \sum_{k=0}^{\infty} \sum_{j \in J_{k}} \omega_{2^{-n-2}}\left(f ; Q_{j}^{k}\right) \chi_{Q_{j}^{k}}(x)
$$

for a.e. $x \in Q_{0}$ [27]. See also [38].

Motivated by this, define the distributional dyadic maximal operator $M_{2^{-n-2}}^{\sharp}$ by

$$
M_{2^{-n-2}}^{\sharp, d} f(x) \equiv \sup _{Q \in \mathcal{D}\left(Q_{0}\right)} \chi_{Q}(x) \omega_{2^{-n-2}}(f ; Q), \quad x \in \mathbf{R}^{n} .
$$

\section{Hardy-Littlewood maximal function - Proof of Theorem 1.2 and Proposition 1.4}

3.1. Proof of Theorem 1.2. Assume that (1) holds. One can deduce the condition

$$
\frac{1}{|R|}\left\|\chi_{Q}\right\|_{L \mathcal{M}_{q}^{p}(u, w)}\left\|\chi_{R}\right\|_{L \mathcal{M}_{q}^{p}(u, w)^{\prime}} \lesssim 1
$$

for all dyadic cubes $Q \in L \mathcal{Q}$ and all cubes $R \in \mathcal{Q}$ with $R \supset 2 Q$ in a well-known manner: simply use

$$
\left(\frac{1}{|R|} \int_{R}|f(y)| \mathrm{d} y\right) \chi_{Q} \leq \inf _{x \in Q} M f(x)
$$


Meanwhile, if $Q \in L \mathcal{Q}$, since we are assuming that $M$ is bounded on $L_{\mathcal{M}}^{p}(u, w)$,

$$
\begin{aligned}
& u\left([-4 \ell(Q), 4 \ell(Q)]^{n}\right)^{\frac{1}{p}-\frac{1}{q}}\left\|\chi_{2 Q} M\left[f \chi_{2 Q}\right]\right\|_{L^{q}(w)} \\
& \lesssim\left\|M\left[f \chi_{2 Q}\right]\right\|_{L \mathcal{M}_{q}^{p}(u, w)} \\
& \lesssim\left\|f \chi_{2 Q}\right\|_{L \mathcal{M}_{q}^{p}(u, w)} \\
& \sim u\left([-\ell(Q) / 2, \ell(Q) / 2]^{n}\right)^{\frac{1}{p}-\frac{1}{q}}\left\|f \chi_{2 Q}\right\|_{L^{q}(w)} .
\end{aligned}
$$

Thus, thanks to the doubling property of $u$ at the origin,

$$
\left\|M\left[f \chi_{2 Q}\right]\right\|_{L^{q}(w)} \lesssim\left\|f \chi_{2 Q}\right\|_{L^{q}(w)} .
$$

This is equivalent to

$$
\sup _{R \in \mathcal{Q}, R \subset 2 Q} \frac{1}{|R|}\left\|\chi_{R}\right\|_{L^{q}(w)}\left\|\chi_{R}\right\|_{L^{q}(w)^{\prime}} \lesssim 1
$$

Let us prove the converse: assume that (2) holds. We have that, for $Q \in L \mathcal{Q}$,

$$
\chi_{Q}(x) M f(x) \leq \chi_{Q}(x) M\left[f \chi_{2 Q}\right](x)+C \sup _{R \in \mathcal{Q}, R \supset 2 Q} \frac{\chi_{Q}(x)}{|R|} \int_{R}|f(y)| \mathrm{d} y .
$$

Together with the $A_{q}$-property at $Q$ this implies

$$
\begin{aligned}
& u(Q)^{\frac{1}{p}-\frac{1}{q}}\left\|\chi_{Q} M f\right\|_{L^{q}(w)} \\
& \lesssim u(Q)^{\frac{1}{p}-\frac{1}{q}}\left\|\chi_{Q} M\left[f \chi_{2 Q}\right]\right\|_{L^{q}(w)}+u(Q)^{\frac{1}{p}-\frac{1}{q}}\left\|\chi_{Q}\right\|_{L^{q}(w)} \sup _{R \in \mathcal{Q}, R \supset 2 Q} \frac{1}{|R|} \int_{R}|f(y)| \mathrm{d} y \\
& \lesssim u\left([-4 \ell(Q), 4 \ell(Q)]^{n}\right)^{\frac{1}{p}-\frac{1}{q}}\left\|f \chi_{2 Q}\right\|_{L^{q}(w)} \\
& \quad+\left\|\chi_{Q}\right\|_{L \mathcal{M}_{q}^{p}(u, w)} \sup _{R \in \mathcal{Q}, R \supset 2 Q} \frac{1}{|R|}\left\|\chi_{R}\right\|_{L \mathcal{M}_{q}^{p}(u, w)^{\prime}}\|f\|_{L \mathcal{M}_{q}^{p}(u, w)} \\
& \lesssim\|f\|_{L \mathcal{M}_{q}^{p}(u, w)},
\end{aligned}
$$

where we have used Lemma 1.1 and the conditions (i) and (ii) of $u$. Thus, the proof is complete.

3.2. Proof of Proposition 1.4. We need only verify that $w \in \mathcal{G}$, if $(1)$ holds. Once this is verified, then we are in the position of using Theorem 1.2.

For $Q=[-R, R]^{n}, R>0$, and any measurable set $E \subset Q$, since

$$
\frac{|E|}{|Q|} w(Q)^{\frac{1}{p}}=w(Q)^{\frac{1}{p}-\frac{1}{q}}\left\|\frac{|E|}{|Q|} \chi_{Q}\right\|_{L^{q}(w)}
$$

$L^{p}(w) \hookrightarrow L \mathcal{M}_{q}^{p}(w, w)$ and $M$ is assumed bounded on $L \mathcal{M}_{q}^{p}(w, w)$, we have that

$$
\frac{|E|}{|Q|} w(Q)^{\frac{1}{p}} \leq\left\|M \chi_{E}\right\|_{L \mathcal{M}_{q}^{p}(w, w)} \lesssim\left\|\chi_{E}\right\|_{L \mathcal{M}_{q}^{p}(w, w)} \leq\left\|\chi_{E}\right\|_{L^{p}(w)}=w(E)^{\frac{1}{p}} .
$$

This $A_{\infty}$-property of $w$ at the origin is more than enough to guarantee that $w \in \mathcal{G}$.

\section{Boundedness of the singular integral operators - Proof of Theorems 1.5, 1.6 and Proposition 1.7}

4.1. Proof of Theorem 1.5. Let $Q_{0} \in L \mathcal{Q}$ be a fixed cube. Form the LernerHytönen decomposition of $T f$ at $Q_{0}$. Then we obtain a sparse family of dyadic cubes 
$\left\{Q_{j}^{k}\right\}_{k \in \mathbf{N}_{0}, j \in J_{k}}$ in $Q_{0}$ satisfying

$$
\left|T f(x)-m_{T f}\left(Q_{0}\right)\right| \leq 2 \sum_{k=0}^{\infty} \sum_{j \in J_{k}} \omega_{2^{-n-2}}\left(T f ; Q_{j}^{k}\right) \chi_{Q_{j}^{k}}(x)
$$

for almost every $x \in Q_{0}$. Thus we have

$$
\begin{aligned}
& u\left(Q_{0}\right)^{\frac{1}{p}-\frac{1}{q}}\left\|w^{\frac{1}{q}} \chi_{Q_{0}} T f\right\|_{L^{q}} \\
& \leq 2 u\left(Q_{0}\right)^{\frac{1}{p}-\frac{1}{q}}\left\|w^{\frac{1}{q}} \sum_{k=0}^{\infty} \sum_{j \in J_{k}} \omega_{2^{-n-2}}\left(T f ; Q_{j}^{k}\right) \chi_{Q_{j}^{k}}\right\|_{L^{q}}+u\left(Q_{0}\right)^{\frac{1}{p}-\frac{1}{q}} w\left(Q_{0}\right)^{\frac{1}{q}}\left|m_{T f}\left(Q_{0}\right)\right| .
\end{aligned}
$$

Let us set $\sigma=w^{-\frac{1}{q-1}}$. To dualize the first term in the right-hand side, we choose a non-negative function $g \in L^{q^{\prime}}(\sigma)$ and consider

$$
\mathrm{I}=\int_{\mathbf{R}^{n}} \sum_{k=0}^{\infty} \sum_{j \in J_{k}} \omega\left(T f ; Q_{j}^{k}\right) \chi_{Q_{j}^{k}}(x) g(x) d x .
$$

Then we have

$$
\begin{aligned}
\mathrm{I} & =\sum_{k=0}^{\infty} \sum_{j \in J_{k}} \int_{\mathbf{R}^{n}} \omega\left(T f ; Q_{j}^{k}\right) \chi_{Q_{j}^{k}}(x) g(x) d x \\
& =\sum_{k=0}^{\infty} \sum_{j \in J_{k}} \omega\left(T f ; Q_{j}^{k}\right) \int_{Q_{j}^{k}} g(x) d x \\
& \leq \sum_{k=0}^{\infty} \sum_{j \in J_{k}} \omega\left(T f ; Q_{j}^{k}\right)\left|Q_{j}^{k}\right| \inf _{x \in Q_{j}^{k}} M g(x) .
\end{aligned}
$$

Let us set $E_{j}^{k}=Q_{j}^{k} \backslash \Omega_{k+1}$. Then we have $2\left|E_{j}^{k}\right| \geq\left|Q_{j}^{k}\right|$. Since $T$ is weak- $(1,1)$ bounded, we have

$$
\mathrm{I} \leq 2 \sum_{k=0}^{\infty} \sum_{j \in J_{k}} \inf _{x \in Q_{j}^{k}} M f(x)\left|E_{j}^{k}\right| \inf _{x \in Q_{j}^{k}} M g(x) .
$$

Since $\left\{E_{j}^{k}\right\}_{k \in \mathbf{N}, j \in J_{k}}$ is a disjoint family contained in $Q_{0}$, we have

$$
\mathrm{I} \leq 2 \int_{Q_{0}} M f(x) M g(x) d x
$$

If we use the $L^{q^{\prime}}(\sigma)$-boundedness of $M$ in $Q_{0}$ and $\|g\|_{L^{q^{\prime}(\sigma)}}=1$, then we have

$$
\left\|w^{\frac{1}{q}} \sum_{k=0}^{\infty} \sum_{j \in J_{k}} \omega_{2^{-n-2}}\left(T f ; Q_{j}^{k}\right) \chi_{Q_{j}^{k}}\right\|_{L^{q}} \lesssim\left\|w^{\frac{1}{q}} M f\right\|_{L^{q}\left(Q_{0}\right)} .
$$

Consequently, since $M$ is bounded thanks to Theorem 1.2,

$$
\begin{aligned}
u\left(Q_{0}\right)^{\frac{1}{p}-\frac{1}{q}}\left\|w^{\frac{1}{q}} \sum_{k=0}^{\infty} \sum_{j \in J_{k}} \omega_{2^{-n-2}}\left(T f ; Q_{j}^{k}\right) \chi_{Q_{j}^{k}}\right\|_{L^{q}} & \lesssim u\left(Q_{0}\right)^{\frac{1}{p}-\frac{1}{q}}\left\|w^{\frac{1}{q}} M f\right\|_{L^{q}\left(Q_{0}\right)} \\
& \lesssim\|M f\|_{L \mathcal{M}_{q}^{p}(u, w)} \\
& \lesssim\|f\|_{L \mathcal{M}_{q}^{p}(u, w) .}
\end{aligned}
$$

Since we are assuming that there exists $\alpha>1$ such that

$$
\left\|\chi_{2^{k+1} Q_{0}}\right\|_{L \mathcal{M}_{q}^{p}(u, w)} \geq \alpha\left\|\chi_{2^{k} Q_{0}}\right\|_{L \mathcal{M}_{q}^{p}(u, w)}
$$


for all $k=0,1,2, \ldots$ and

$$
\left|m_{T f}\left(Q_{0}\right)\right| \lesssim \sum_{l=1}^{\infty} \frac{1}{\left|2^{l} Q_{0}\right|} \int_{2^{l} Q_{0}}|f(x)| \mathrm{d} x
$$

we have

$$
\begin{aligned}
\left|m_{T f}(Q)\right| \cdot u\left(Q_{0}\right)^{\frac{1}{p}-\frac{1}{q}} w\left(Q_{0}\right)^{\frac{1}{q}} & \lesssim \sum_{l=1}^{\infty} \alpha^{-l} u\left(2^{l} Q_{0}\right)^{\frac{1}{p}-\frac{1}{q}} w\left(2^{l} Q_{0}\right)^{\frac{1}{q}} \frac{1}{\left|2^{l} Q_{0}\right|} \int_{2^{l} Q_{0}}|f(x)| \mathrm{d} x \\
& \lesssim\|M f\|_{L \mathcal{M}_{q}^{p}(u, w)} \\
& \lesssim\|f\|_{L \mathcal{M}_{q}^{p}(u, w)}
\end{aligned}
$$

as was to be shown.

4.2. Proof of Theorem 1.6. We say that a sequence $Q_{0}, Q_{1}, \ldots, Q_{K}$ in $L \mathcal{Q}$ is a chain if $\ell\left(Q_{k-1}\right)=2 \ell\left(Q_{k}\right)$ and $\overline{Q_{k-1}}$ and $\overline{Q_{k}}$ intersect at a set of Lebesgue measure zero for all $k=1,2, \ldots, K$ and $Q_{j} \cap Q_{j}=\emptyset$ if $|j-k| \geq 1$. We need a lemma.

Lemma 4.1. Let $1<q<p<\infty$ and let $w$ be a weight. Assume that $u \in \mathcal{G}$ and that $\left\|R_{j} f\right\|_{L \mathcal{M}_{q}^{p}(u, w)} \lesssim\|f\|_{L \mathcal{M}_{q}^{p}(u, w)}$ for all $f \in L_{\mathrm{c}}^{\infty}$ and $j=1,2, \ldots, n$. Suppose that we are given a chain $Q_{0}, Q_{1}, Q_{2}, Q_{3}$ in $L \mathcal{Q}$.

(1) The cubes $\overline{2 Q_{0}}$ and $\overline{Q_{3}}$ do not intersect.

(2) For any non-negative $f \in L_{\mathrm{c}}^{\infty}$ supported on $2 Q_{0}$, we have

$$
\sum_{j=1}^{n}\left|R_{j} f(x)\right| \gtrsim \frac{1}{\left|Q_{0}\right|} \int_{2 Q_{0}} f(y) \mathrm{d} y \quad\left(x \in Q_{3}\right) .
$$

(3) $\left\|\chi_{2 Q_{0}}\right\|_{L \mathcal{M}_{q}^{p}(u, w)} \sim\left\|\chi_{Q_{3}}\right\|_{L \mathcal{M}_{q}^{p}(u, w)}$.

(4) There exists a constant $C>0$ independent of $Q_{0} \in L \mathcal{Q}$ such that

$$
\frac{1}{\left|Q_{0}\right|}\left\|\chi_{2 Q_{0}}\right\|_{L^{q}(w)}\left\|\chi_{2 Q_{0}}\right\|_{L^{q}(w)^{\prime}} \leq C .
$$

Proof. We suppose that $Q_{0}=\left[2^{m}, 2^{m+1}\right)^{n}$ and $Q_{3}=\left[2^{m-3}, 2^{m-2}\right)^{n}$ for the sake of simplicity.

(1) A geometric observation shows that $\overline{2 Q_{0}}=\left[2^{m-1}, 5 \cdot 2^{m-1}\right]^{n}$ and $\overline{Q_{3}}=\left[2^{m-3}\right.$, $\left.2^{m-2}\right]^{n}$, so that $\overline{2 Q_{0}}$ and $\overline{Q_{3}}$ do not intersect.

(2) Let $f \in L_{\mathrm{c}}^{\infty}\left(2 Q_{0}\right)$ be a non-negative function. Since

$$
-\sum_{j=1}^{n} \frac{x_{j}-y_{j}}{|x-y|^{n+1}} \sim \frac{1}{\ell\left(Q_{0}\right)^{n}}
$$

for any $x \in Q_{3}$ and $y \in 2 Q_{0}$, we have

$$
\sum_{j=1}^{n}\left|R_{j} f(x)\right|=-\sum_{j=1}^{n} R_{j} f(x)=-\sum_{j=1}^{n} \int_{\mathbf{R}^{n}} \frac{x_{j}-y_{j}}{|x-y|^{n+1}} f(y) d y \gtrsim \frac{1}{\left|Q_{0}\right|} \int_{2 Q_{0}} f(y) \mathrm{d} y .
$$

(3) By considering $f=\chi_{2 Q_{0}}$ in (2) and using $\left\|R_{j} \chi_{2 Q_{0}}\right\|_{L \mathcal{M}_{q}^{p}(u, w)} \lesssim\left\|\chi_{2 Q_{0}}\right\|_{L \mathcal{M}_{q}^{p}(u, w)}$, we can show that $\left\|\chi_{Q_{3}}\right\|_{L \mathcal{M}_{q}^{p}(u, w)} \gtrsim\left\|\chi_{2 Q_{0}}\right\|_{L \mathcal{M}_{q}^{p}(u, w)}$. We can swap the role of $2 Q_{0}$ and $Q_{3}$ to have $\left\|\chi_{2 Q_{0}}\right\|_{L \mathcal{M}_{q}^{p}(u, w)} \lesssim\left\|\chi_{Q_{3}}\right\|_{L \mathcal{M}_{q}^{p}(u, w)}$ if we go through a similar argument.

(4) From (2) we have

$$
\left\|\chi_{Q_{0}}\right\|_{L \mathcal{M}_{q}^{p}(u, w)} \frac{1}{\left|Q_{0}\right|} \int_{2 Q_{0}} f(y) \mathrm{d} y \lesssim\|f\|_{L \mathcal{M}_{q}^{p}(u, w)} \sim u\left(Q_{0}\right)^{\frac{1}{p}-\frac{1}{q}}\|f\|_{L^{q}(w)}
$$


for any non-negative measurable function $f$ supported on $2 Q_{0}$. It remains to use the duality.

Going through a similar argument, we have the following corollary.

Corollary 4.2. Let $1<q<p<\infty$ and let $w$ be a weight. Assume that $u \in \mathcal{G}$ and that $\left\|R_{j} f\right\|_{L \mathcal{M}_{q}^{p}(u, w)} \lesssim\|f\|_{L \mathcal{M}_{q}^{p}(u, w)}$ for all $f \in L_{\mathrm{c}}^{\infty}$ and $j=1,2, \ldots, n$. Then there exists a constant $C>0$ independent of $Q \in L \mathcal{Q}$ such that

$$
\sup _{R \in \mathcal{Q}, R \subset 2 Q} \frac{1}{|R|}\left\|\chi_{R}\right\|_{L^{q}(w)}\left\|\chi_{R}\right\|_{L^{q}(w)^{\prime}} \leq C .
$$

Proof. Let $R \in \mathcal{Q}$ satisfy $R \subset 2 Q$. By decomposing $R$ or by expanding $R$, we can assume that $R$ is a dyadic cube. Then we can choose a sequence $S=S_{1}, S_{2}, S_{3}=R$ of cubes such that $\ell(R) \sim \ell\left(S_{k}\right) \sim \operatorname{dist}(R, S)$ for $k=1,2,3$, that $S_{k}$ and $S_{k+1}$ intersect at a point for $k=1,2$ and that $2 S_{1} \cap 2 S_{3}=\emptyset$. If we replace $Q_{3}$ by $S$ and $Q_{0}$ by $R$, respectively in the proof of Lemma 4.1, we can argue as before.

We move on to the proof of Theorem 1.6. In view of Corollary 4.2, it remains to show that there exists a constant $C$ which is independent of $Q \in L \mathcal{Q}$ such that

$$
\sup _{R \in \mathcal{Q}, R \supset Q} \frac{1}{|R|}\left\|\chi_{Q}\right\|_{L \mathcal{M}_{q}^{p}(u, w)}\left\|\chi_{R}\right\|_{L \mathcal{M}_{q}^{p}(u, w)^{\prime}} \leq C
$$

for all cubes $Q \in L \mathcal{Q}$. By expanding $R$ we can assume that $R \supset 2 Q$. By decomposing $R$ suitably, we can further assume that there exists a chain $Q=Q_{0}, Q_{1}, Q_{2}, Q_{3}$ such that $R$ and $2 Q_{3}$ do not intersect.

Then for any non-negative function $f \in L^{\infty}$ supported on $R$, we have

$$
\frac{1}{|R|} \int_{R} f(x) \mathrm{d} x \lesssim \sum_{j=1}^{n}\left|R_{j} f(y)\right|,
$$

for all $y \in Q_{3}$ As a result, assuming that $\left\|R_{j} f\right\|_{L \mathcal{M}_{q}^{p}(u, w)} \lesssim\|f\|_{L \mathcal{M}_{q}^{p}(u, w)}$, we see

$$
\left(\frac{1}{|R|} \int_{R} f(x) \mathrm{d} x\right)\left\|\chi_{Q_{3}}\right\|_{L \mathcal{M}_{q}^{p}(u, w)} \lesssim\|f\|_{L \mathcal{M}_{q}^{p}(u, w)} .
$$

Since $\left\|\chi_{Q_{3}}\right\|_{L \mathcal{M}_{q}^{p}(u, w)} \sim\left\|\chi_{Q}\right\|_{L \mathcal{M}_{q}^{p}(u, w)}$ thanks to Lemma 4.1(3), we obtain

$$
\left(\frac{1}{|R|} \int_{R} f(x) \mathrm{d} x\right)\left\|\chi_{Q}\right\|_{L \mathcal{M}_{q}^{p}(u, w)} \lesssim\|f\|_{L \mathcal{M}_{q}^{p}(u, w)} .
$$

By passing to the Köthe dual, we obtain $\left\|\chi_{R}\right\|_{L \mathcal{M}_{q}^{p}(u, w)^{\prime}}\left\|\chi_{Q}\right\|_{L \mathcal{M}_{q}^{p}(u, w)} \lesssim|R|$, as required.

4.3. Proof of Proposition 1.7. We need to prove that $w \in \mathcal{G}$ in particular, $w$ is reverse doubling at the origin. To this end, we observe

$$
\left\|R_{j} f\right\|_{L \mathcal{M}_{q}^{p}(w, w)} \lesssim\|f\|_{L^{p}(w)}
$$

for all $f \in L_{\mathrm{c}}^{\infty}$. Let $Q$ be a cube centered at the origin and let $Q^{\prime}$ be a cube such that $|Q|=\left|Q^{\prime}\right|$ and $\sharp\left(Q \cap Q^{\prime}\right)=1$, where $\sharp E$ stands for the cardinality of the set $E \subset \mathbf{R}^{n}$. Then since $w$ is doubling, we have

$$
w(Q)^{\frac{1}{p}} \frac{1}{|Q|} \int_{Q}|f(y)| \mathrm{d} y \sim w\left(Q^{\prime}\right)^{\frac{1}{p}} \frac{1}{|Q|} \int_{Q}|f(y)| \mathrm{d} y \lesssim\|f\|_{L^{p}(w)}
$$

for all $f \in L^{\infty}$ with $\operatorname{supp}(f) \subset Q$. Let $R$ be a cube contained in $Q$. If we let $f=w^{-\frac{1}{q}} \chi_{R}$, then we obtain $w \in A_{q+1}$. Thus, we see that $w \in \mathcal{G}$ and we are in the position of using Theorem 1.6. 


\section{Fractional integral operators - Proof of Theorem 1.12}

We can prove that (2) and (3) are equivalent similar to Theorem 1.2. Meanwhile, (1) is clearly stronger than (2). It remains to show that (3) implies (1) under an additional assumption. This is achieved similar to Proposition 1.8 using $M_{2^{-n-2}}^{\sharp}\left(I_{\alpha} f\right)(x) \lesssim M_{\alpha} f(x),\left(x \in \mathbf{R}^{n}\right)$.

\section{The case of the power weight - Proof of Propositions 1.8 and $1.9,1.13$ and 1.14}

Here we consider the case where $w(x)=|x|^{\beta}$ where $\beta>-n$. Note that $w \in A_{\infty}$, so that $w \in \mathcal{G}$.

6.1. Proof of Proposition 1.8. Let us assume $-\frac{q}{p} n \leq \beta<q\left(1-\frac{1}{p}\right) n$. It is clear that

$$
\sup _{R \in \mathcal{D}, R \subset Q} \frac{1}{|R|}\left\|\chi_{R}\right\|_{L^{q}(w)}\left\|\chi_{R}\right\|_{L^{q}(w)^{\prime}} \lesssim \sup _{R \in \mathcal{D}, R \subset Q} \frac{1}{|R|}\left\|\chi_{R} w\left(c_{Q}\right)^{\frac{1}{q}}\right\|_{L^{q}}\left\|\chi_{R} w\left(c_{Q}\right)^{-\frac{1}{q}}\right\|_{L^{q^{\prime}}}=1
$$

for all cubes $Q \in L \mathcal{Q}$. Next we will prove

$$
\left\|\chi_{Q}\right\|_{L^{q}(w)}\left\|\chi_{R}\right\|_{L \mathcal{M}_{q}^{p}(1, w)^{\prime}} \leq C|Q|^{\frac{1}{q}-\frac{1}{p}}|R|
$$

or equivalently

$$
\left\|\chi_{Q}\right\|_{L \mathcal{M}_{q}^{p}(1, w)}\left\|\chi_{R}\right\|_{L \mathcal{M}_{q}^{p}(1, w)^{\prime}} \lesssim|R|
$$

for all cubes $Q \in L \mathcal{Q}$ and $R$ such that $Q \subset R$. To this end, by replacing $R$ with a larger one, say $10 R$, we may assume that $R=[-2 r, 2 r]^{n}$ is centered at the origin. Write $R^{*}=[r, 2 r]^{n}$. By the dilation formula for $\left(L \mathcal{M}_{q}^{p}\right)^{\prime}$, we have

$$
\left\|\chi_{R^{*}}\left(2^{l} \cdot\right) w^{-\frac{1}{p}}\right\|_{\left(L \mathcal{M}_{q}^{p}\right)^{\prime}}=2^{\frac{l \beta}{q}}\left\|\chi_{R^{*}}\left(2^{l} \cdot\right) w\left(2^{l} \cdot\right)^{-\frac{1}{q}}\right\|_{\left(L \mathcal{M}_{q}^{p}\right)^{\prime}}=2^{\frac{l \beta}{q}-\frac{l n}{p^{\prime}}}\left\|\chi_{R^{*}} \cdot w^{-\frac{1}{q}}\right\|_{\left(L \mathcal{M}_{q}^{p}\right)^{\prime}}
$$

Then since $\beta<q\left(1-\frac{1}{p}\right) n$, we have

$$
\begin{aligned}
\left\|\chi_{R}\right\|_{L \mathcal{M}_{q}^{p}(1, w)^{\prime}} & =\left\|\chi_{R} w^{-\frac{1}{q}}\right\|_{\left(L \mathcal{M}_{q}^{p}\right)^{\prime}} \leq \sum_{l=0}^{\infty}\left\|\chi_{2^{-l} R \backslash 2^{-l-1} R} w^{-\frac{1}{q}}\right\|_{\left(L \mathcal{M}_{q}^{p}\right)^{\prime}} \\
& \sim \sum_{l=0}^{\infty}\left\|\chi_{R^{*}}\left(2^{l} \cdot\right) w^{-\frac{1}{q}}\right\|_{\left(L \mathcal{M}_{q}^{p}\right)^{\prime}} \sim\left\|\chi_{R^{*}} w^{-\frac{1}{q}}\right\|_{\left(L \mathcal{M}_{q}^{p}\right)^{\prime}} \\
& \sim w\left(c_{R^{*}}\right)^{-\frac{1}{q}}\left\|\chi_{R^{*}}\right\|_{\left(L \mathcal{M}_{q}^{p}\right)^{\prime}} .
\end{aligned}
$$

Thus, since $\frac{q}{p} n+\beta>0$, thanks to [44, Example 2.3]

$$
\begin{aligned}
\left\|\chi_{Q}\right\|_{L^{q}(w)}\left\|\chi_{R}\right\|_{L \mathcal{M}_{q}^{p}(1, w)^{\prime}} & \lesssim w\left(c_{Q}\right)^{\frac{1}{q}}\left\|\chi_{Q}\right\|_{L^{q}} w\left(c_{R^{*}}\right)^{-\frac{1}{q}}\left\|\chi_{R^{*}}\right\|_{\left(L \mathcal{M}_{q}^{p}\right)^{\prime}} \\
& \lesssim \ell(Q)^{\frac{n+\beta}{q}} w\left(c_{R^{*}}\right)^{-\frac{1}{q}}|R|^{\frac{1}{p^{\prime}}} \lesssim \ell(Q)^{\frac{n+\beta}{q}} \ell(R)^{n-\frac{n+\beta}{p}} \\
& \lesssim|Q|^{\frac{1}{q}-\frac{1}{p}}|R| .
\end{aligned}
$$

Let us assume that $M$ is bounded on $\operatorname{LM}_{q}^{p}\left(1,|x|^{\beta}\right)$. Then $\chi_{[-1,1]^{n}} \in \operatorname{LM}_{q}^{p}\left(1, w_{\beta}\right)$ and $\frac{\chi_{B}|\cdot|^{-n}}{\log |\cdot|} \notin L \mathcal{M}_{q}^{p}\left(1, w_{\beta}\right)$, where $B$ is a small open ball centered at the origin. We observe that $\chi_{[-1,1]^{n}} \in L \mathcal{M}_{q}^{p}\left(1, w_{\beta}\right)$ if and only if $\beta \geq-\frac{q}{p} n$ and that $\frac{\chi_{B}|\cdot|^{-n}}{\log |\cdot|} \notin$ $L \mathcal{M}_{q}^{p}\left(1, w_{\beta}\right)$ if and only if $\beta<q\left(1-\frac{1}{p}\right) n$. 
6.2. Proof of Proposition 1.9. According to [6], (2) and (3) are equivalent. If we assume (1), then $M$ is bounded from $L^{p}(w)$ to $L \mathcal{M}_{q}^{p}(w, w)$. As a result,

$$
w(Q)^{\frac{1}{p}}\left(\frac{1}{|Q|} \int_{Q}|f(y)| \mathrm{d} y\right) \leq C\|f\|_{L^{p}(w)}
$$

for all measurable functions $f$ and all cubes centered at the origin. Thus,

$$
\frac{w(Q)}{|Q|} \cdot\left(\frac{\sigma(Q)}{|Q|}\right)^{p-1} \lesssim 1
$$

for all cubes centered at the origin, where $\sigma(x)=w(x)^{-\frac{1}{p-1}}, x \in \mathbf{R}^{n}$. As a consequence we have $-n<\beta<n(p-1)$.

Assume $-n<\beta<n(p-1)$. As before, it is clear that

$\sup _{R \in \mathcal{D}, R \subset Q} \frac{1}{|R|}\left\|\chi_{R}\right\|_{L^{q}(w)}\left\|\chi_{R}\right\|_{L^{q}(w)^{\prime}} \leq C \sup _{R \in \mathcal{D}, R \subset Q} \frac{1}{|R|}\left\|\chi_{R} w\left(c_{Q}\right)^{\frac{1}{q}}\right\|_{L^{q}}\left\|\chi_{R} w\left(c_{Q}\right)^{-\frac{1}{q}}\right\|_{L^{q^{\prime}}}=C$ for all cubes $Q \in L \mathcal{Q}$.

Next we will establish

$$
\left\|\chi_{Q}\right\|_{L^{q}(w)}\left\|\chi_{R}\right\|_{L \mathcal{M}_{q}^{p}(w, w)^{\prime}} \leq C w(Q)^{\frac{1}{q}-\frac{1}{p}}|R|
$$

or equivalently

$$
\left\|\chi_{Q}\right\|_{L \mathcal{M}_{q}^{p}(w, w)}\left\|\chi_{R}\right\|_{L \mathcal{M}_{q}^{p}(w, w)^{\prime}} \lesssim|R|
$$

for all cubes $Q \in L \mathcal{Q}$ and $R$ such that $Q \subset R$. To this end, by replacing $R$ with a larger one, say $Q(0,10 \ell(R))$, we may assume that $R=[-2 r, 2 r]^{n}$ is centered at the origin. Write $R^{*}=[r, 2 r]^{n}$. By the dilation formula for $L_{\mathcal{M}}^{p}(w, w)^{\prime}$, we have

$$
\left\|\chi_{R^{*}}\left(2^{l} \cdot\right)\right\|_{L \mathcal{M}_{q}^{p}(w, w)^{\prime}}=2^{-l n+\frac{l(n+\beta)}{p}}\left\|\chi_{R^{*}}\right\|_{L \mathcal{M}_{q}^{p}(w, w)^{\prime}}
$$

Since $w$ is a power weight

$$
\left\|\chi_{2^{-l} R \backslash 2^{-l-1} R}\right\|_{L \mathcal{M}_{q}^{p}(w, w)^{\prime}} \sim\left\|\chi_{R^{*}}\left(2^{l} \cdot\right)\right\|_{L \mathcal{M}_{q}^{p}(w, w)^{\prime}}
$$

Thus,

$$
\left\|\chi_{R}\right\|_{L \mathcal{M}_{q}^{p}(w, w)^{\prime}} \leq \sum_{l=0}^{\infty}\left\|\chi_{2^{-l} R \backslash 2^{-l-1} R}\right\|_{L \mathcal{M}_{q}^{p}(w, w)^{\prime}} \lesssim \sum_{l=0}^{\infty}\left\|\chi_{R^{*}}\left(2^{l} \cdot\right)\right\|_{L \mathcal{M}_{q}^{p}(w, w)^{\prime}}
$$

Assuming $\beta<n(p-1)$, we have

$$
\begin{aligned}
\left\|\chi_{R}\right\|_{L \mathcal{M}_{q}^{p}(w, w)^{\prime}} & \lesssim \sum_{l=0}^{\infty} 2^{-\ln +\frac{l(n+\beta)}{p}}\left\|\chi_{R^{*}}\right\|_{L \mathcal{M}_{q}^{p}(w, w)^{\prime}} \sim\left\|\chi_{R^{*}}\right\|_{L \mathcal{M}_{q}^{p}(w, w)^{\prime}} \\
& \lesssim w\left(R^{*}\right)^{\frac{1}{q}-\frac{1}{p}} w\left(c_{R^{*}}\right)^{-\frac{1}{q}}\left|R^{*}\right|^{\frac{1}{q^{\prime}}} \lesssim w\left(R^{*}\right)^{-\frac{1}{p}}\left|R^{*}\right| .
\end{aligned}
$$

As a result, since $n+\beta>0$, thanks to [44, Example 2.3]

$$
\begin{aligned}
&\left\|\chi_{Q}\right\|_{L^{q}(w)}\left\|\chi_{R}\right\|_{L \mathcal{M}_{q}^{p}(w, w)^{\prime}} \lesssim w(Q)^{\frac{1}{q}} w\left(R^{*}\right)^{-\frac{1}{p}}\left|R^{*}\right| \lesssim \ell(Q)^{\frac{n+\beta}{q}} \ell(R)^{n-\frac{n+\beta}{p}} \\
& \lesssim \ell(Q)^{\frac{n+\beta}{q}-\frac{n+\beta}{p}}|R| \lesssim w(Q)^{\frac{1}{q}-\frac{1}{p}}|R| .
\end{aligned}
$$

Thus (6.1) is proved.

6.3. Proof of Proposition 1.11. (1) is sufficient for (2) and (3) similar to Proposition 1.8. Let $Q$ be a cube. We need to show that

$$
w(Q)^{\frac{1}{p}-\frac{1}{q}}\left(\int_{Q} M f(x)^{q} w(x) \mathrm{d} x\right)^{\frac{1}{q}} \lesssim\|f\|_{L \mathcal{M}_{q}^{p}(w, w)}
$$


for all cubes $Q$. If $-n<\beta<n(q-1)$, then we can go through the same argument as [37] which is followed by $[12,40,56,59]$ and so on. So we assume $\beta \geq n(q-1)$. We may assume that $Q$ is centered at the origin or that $0 \notin 4 Q$. Let $\varepsilon \in\left(0, \frac{q}{p}(n(p-1)-\beta)\right)$. We distinguish four cases:

(1) Assume that $f$ is supported on $3 Q$ and that $Q$ is centered at the origin. We borrow the idea of [7]. In this case, we have

$$
w(Q)=w_{\beta}(Q) \sim \ell(Q)^{n+\beta}
$$

from [44, Example 2.3] and

$$
\left(\int_{Q} M f(x)^{q} w(x) \mathrm{d} x\right)^{\frac{1}{q}} \lesssim \ell(Q)^{\frac{\beta-n(q-1)+\varepsilon}{q}}\left(\int_{Q} M f(x)^{q}|x|^{n(q-1)-\varepsilon} \mathrm{d} x\right)^{\frac{1}{q}}
$$

since $\beta \geq n(q-1)>n(q-1)-\varepsilon$. Since $|x|^{n(q-1)-\varepsilon} \in A_{q}$, we have

$$
\left(\int_{Q} M f(x)^{q}|x|^{n(q-1)-\varepsilon} \mathrm{d} x\right)^{\frac{1}{q}} \lesssim\left(\int_{3 Q}|f(x)|^{q}|x|^{n(q-1)-\varepsilon} \mathrm{d} x\right)^{\frac{1}{q}} .
$$

We next decompose $3 Q$ dyadically to have

$$
\begin{aligned}
& \left(\int_{3 Q}|f(x)|^{q}|x|^{n(q-1)-\varepsilon} \mathrm{d} x\right)^{\frac{1}{q}} \\
& \leq \sum_{l=0}^{\infty}\left(\int_{3 \cdot 2^{-l} Q \backslash 3 \cdot 2^{-l-1} Q}|f(x)|^{q}|x|^{n(q-1)-\varepsilon} \mathrm{d} x\right)^{\frac{1}{q}} \\
& \sim \sum_{l=0}^{\infty}\left(2^{-l} \ell(Q)\right)^{\frac{n(q-1)-\varepsilon-\beta}{q}}\left(\int_{3 \cdot 2^{-l} Q \backslash 3 \cdot 2^{-l-1} Q}|f(x)|^{q}|x|^{\beta} \mathrm{d} x\right)^{\frac{1}{q}} \\
& \lesssim \sum_{l=0}^{\infty}\left(2^{-l} \ell(Q)\right)^{\frac{n(q-1)-\varepsilon-\beta}{q}}\left(2^{-l} \ell(Q)\right)^{\frac{n+\beta}{q}-\frac{n+\beta}{p}}\|f\|_{\mathcal{M}_{q}^{p}\left(1, w_{\beta}\right)} .
\end{aligned}
$$

Arithmetic shows that

$$
\frac{n(q-1)-\varepsilon-\beta}{q}+\frac{n+\beta}{q}-\frac{n+\beta}{p}=\frac{n(p-1)-\beta}{p}-\frac{\varepsilon}{q}>0 .
$$

Thus, the series in the most right-hand side converges to have

$$
\ell(Q)^{\frac{n+\beta}{p}-\frac{n+\beta}{q}} \ell(Q)^{\frac{\beta-n(q-1)+\varepsilon}{q}}\left(\int_{3 Q}|f(x)|^{q}|x|^{n(q-1)-\varepsilon} \mathrm{d} x\right)^{\frac{1}{q}} \lesssim\|f\|_{\mathcal{M}_{q}^{p}(w, w)} .
$$

Consequently, we have (6.2).

(2) Assume that $f$ is supported outside $3 Q$ and that $Q$ is centered at the origin.

In this case, using [44, Example 2.3] again, we have

$$
\begin{aligned}
w(Q)^{\frac{1}{p}-\frac{1}{q}}\left(\int_{Q} M f(x)^{q} w(x) \mathrm{d} x\right)^{\frac{1}{q}} & \lesssim \ell(Q)^{\frac{n+\beta}{p}} \sup _{R>\ell(Q)} \frac{1}{|Q(R)|} \int_{Q(R)}|f(y)| \mathrm{d} y \\
& \lesssim \sup _{l \in \mathbf{N}} \ell(Q)^{\frac{n+\beta}{p}} \frac{1}{\left|2^{l} Q\right|} \int_{2^{l} Q \backslash Q}|f(y)| \mathrm{d} y .
\end{aligned}
$$

Let $l \in \mathbf{N}$ be fixed. We decompose $2^{l} Q \backslash Q$ dyadically to have

$$
\ell(Q)^{\frac{n+\beta}{p}} \frac{1}{\left|2^{l} Q\right|} \int_{2^{l} Q \backslash Q}|f(y)| \mathrm{d} y=\ell(Q)^{\frac{n+\beta}{p}} \sum_{k=1}^{l} \frac{1}{\left|2^{l} Q\right|} \int_{2^{k} Q \backslash 2^{k-1} Q}|f(y)| \mathrm{d} y
$$


If we use the Hölder inequality, then we have

$$
\begin{aligned}
& \ell(Q)^{\frac{n+\beta}{p}} \frac{1}{\left|2^{l} Q\right|} \int_{2^{l} Q \backslash Q}|f(y)| \mathrm{d} y \\
& \leq \ell(Q)^{\frac{n+\beta}{p}} \sum_{k=1}^{l} 2^{n(k-l)}\left(\frac{1}{\left|2^{k} Q\right|} \int_{2^{k} Q \backslash 2^{k-1} Q}|f(y)|^{q} \mathrm{~d} y\right)^{\frac{1}{q}} \\
& \lesssim \ell(Q)^{\frac{n+\beta}{p}-\frac{\beta}{q}} \sum_{k=1}^{l} 2^{n(k-l)-\frac{\beta}{q} k}\left(\frac{1}{\left|2^{k} Q\right|} \int_{2^{k} Q \backslash 2^{k-1} Q}|f(y)|^{q}|y|^{\beta} \mathrm{d} y\right)^{\frac{1}{q}} \\
& \lesssim \sum_{k=1}^{l} 2^{n(k-l)-\frac{n+\beta}{p} k}\|f\|_{\mathcal{M}_{q}^{p}(w, w) .}
\end{aligned}
$$

Since $\sum_{k=1}^{l} 2^{n(k-l)-\frac{n+\beta}{p} k} \lesssim 1$, we obtain (6.2).

(3) Assume that $f$ is supported on $3 Q$ and that $0 \notin 4 Q$. Then we have

$$
\begin{aligned}
& w(Q)^{\frac{1}{p}-\frac{1}{q}}\left(\int_{Q} M f(x)^{q} w(x) \mathrm{d} x\right)^{\frac{1}{q}} \\
& \sim(|Q| w(c(Q)))^{\frac{1}{p}-\frac{1}{q}}\left(\int_{Q} M f(x)^{q} w(c(Q)) \mathrm{d} x\right)^{\frac{1}{q}} \\
& \lesssim(|Q| w(c(Q)))^{\frac{1}{p}-\frac{1}{q}}\left(\int_{3 Q}|f(x)|^{q} w(c(Q)) \mathrm{d} x\right)^{\frac{1}{q}} \\
& \lesssim w(Q)^{\frac{1}{p}-\frac{1}{q}}\left(\int_{3 Q}|f(x)|^{q} w(x) \mathrm{d} x\right)^{\frac{1}{q}} .
\end{aligned}
$$

(4) Assume that $f$ is supported outside $3 Q$ and that $0 \notin 4 Q$. Then we have

$$
\begin{aligned}
& w(Q)^{\frac{1}{p}-\frac{1}{q}}\left(\int_{Q} M f(x)^{q} w(x) \mathrm{d} x\right)^{\frac{1}{q}} \\
& \sim(|Q| w(c(Q)))^{\frac{1}{p}-\frac{1}{q}}\left(\int_{Q} M f(x)^{q} w(c(Q)) \mathrm{d} x\right)^{\frac{1}{q}} \\
& \sim(|Q| w(c(Q)))^{\frac{1}{p}} \sup _{R: \text { cubes }, R \supset Q} \frac{1}{|R|} \int_{R \backslash 3 Q}|f(x)| \mathrm{d} x \\
& \sim(|Q| w(c(Q)))^{\frac{1}{p}} \sup _{R: \text { cubes }, R \supset Q, c(Q)=c(R)} \frac{1}{|R|} \int_{R \backslash 3 Q}|f(x)| \mathrm{d} x .
\end{aligned}
$$

If $0 \notin 8 R, c(Q)=c(R)$ and $Q \subset R$, then we have

$$
\begin{aligned}
& (|Q| w(c(Q)))^{\frac{1}{p}} \frac{1}{|R|} \int_{R \backslash 3 Q}|f(x)| \mathrm{d} x \\
& \lesssim(|R| w(c(R)))^{\frac{1}{p}} \frac{1}{w(R)} \int_{R \backslash 3 Q}|f(x)| w(x) \mathrm{d} x \\
& \lesssim w(R)^{\frac{1}{p}}\left(\frac{1}{w(R)} \int_{R \backslash 3 Q}|f(x)|^{q} w(x) \mathrm{d} x\right)^{\frac{1}{q}} \leq\|f\|_{L \mathcal{M}_{q}^{p}(w, w)} .
\end{aligned}
$$


Suppose instead that $0 \in 8 R, c(Q)=c(R)$ and $Q \subset R$. Then we have

$$
\begin{aligned}
\frac{1}{|R|} \int_{R \backslash 3 Q}|f(x)| \mathrm{d} x & \leq \frac{1}{|R|}\left(\int_{R}|f(x)|^{q}|x|^{n(q-1)-\varepsilon} \mathrm{d} x\right)^{\frac{1}{q}}\left(\int_{R}|x|^{-n+\frac{\varepsilon}{q-1}} \mathrm{~d} x\right)^{\frac{1}{q^{\prime}}} \\
& \lesssim \frac{1}{|R|} \ell(R)^{\frac{\varepsilon}{q}}\left(\int_{R}|f(x)|^{q}|x|^{n(q-1)-\varepsilon} \mathrm{d} x\right)^{\frac{1}{q}} .
\end{aligned}
$$

From (6.3) we have

$$
\left(\int_{R}|f(x)|^{q}|x|^{n(q-1)-\varepsilon} \mathrm{d} x\right)^{\frac{1}{q}} \lesssim \ell(R)^{\frac{\beta-n(q-1)-\varepsilon}{q}} w(R)^{-\frac{1}{p}+\frac{1}{q}}\|f\|_{\mathcal{M}_{q}^{p}(w, w)} .
$$

As a result,

$$
w(Q)^{\frac{1}{p}} \frac{1}{|R|} \int_{R \backslash 3 Q}|f(x)| \mathrm{d} x \lesssim w(R)^{\frac{1}{p}} \ell(R)^{\frac{\beta+n}{q}} w(R)^{-\frac{1}{p}+\frac{1}{q}}\|f\|_{\mathcal{M}_{q}^{p}(w, w)} \lesssim\|f\|_{\mathcal{M}_{q}^{p}(w, w)} .
$$

Putting these results all together, we obtain the desired result.

6.4. Proof of Proposition 1.13. If we assume (1), then we have $\chi_{[-1,1]^{n}} \in$ $L \mathcal{M}_{t}^{s}\left(1, w^{t}\right)$ and $\chi_{B}|\cdot|^{-n} \notin L \mathcal{M}_{q}^{p}\left(1, w^{q}\right)$, or equivalently $\chi_{B}|\cdot|^{-n+\beta} \notin L \mathcal{M}_{q}^{p}$ where $B$ is a small open ball centered at the origin. We observe that $\chi_{[-1,1]^{n}} \in L \mathcal{M}_{t}^{s}\left(1, w^{t}\right)$ if and only if $\beta \geq-\frac{n}{s}$, and that $\chi_{B}|\cdot|^{-n+\beta} \notin L \mathcal{M}_{q}^{p}$, if and only if $\beta-n<-\frac{n}{p}$. If we assume $-\frac{n}{s} \leq \beta<\frac{n}{p^{\prime}}$, then we can argue as we did in the proof of Proposition 1.8, to conclude that $w=w_{\beta}$ satisfies

$\sup _{R \in \mathcal{Q}, R \subset 2 Q} \frac{\ell(R)^{\beta}}{|R|}\left\|\chi_{R}\right\|_{L^{t}\left(w^{t}\right)}\left\|\chi_{R}\right\|_{L^{q}\left(w^{q}\right)^{\prime}}+\sup _{R \in \mathcal{Q}, R \supset Q} \frac{\ell(R)^{\beta}}{|R|}\left\|\chi_{Q}\right\|_{L \mathcal{M}_{t}^{s}\left(1, w^{t}\right)}\left\|\chi_{R}\right\|_{L \mathcal{M}_{q}^{p}\left(1, w^{q}\right)^{\prime}} \leq C$ for all cubes $Q \in L \mathcal{Q}$.

In fact, if $R$ is a cube such that $R \subset 2 Q$, then we have

$$
\frac{\ell(R)^{\beta}}{|R|}\left\|\chi_{R}\right\|_{L^{t}\left(w^{t}\right)}\left\|\chi_{R}\right\|_{L^{q}\left(w^{q}\right)^{\prime}} \sim \frac{\ell(R)^{\beta}}{|R|}\left\|\chi_{R}\right\|_{L^{t}}\left\|\chi_{R}\right\|_{\left(L^{q}\right)^{\prime}}=1 .
$$

Meanwhile if $R$ is a cube such that $R \supset Q$, we denote by $R^{*}$ the cube satisfying $\left|R^{*}\right|=100^{n}|R|$ and centered at the origin. Then

$$
\frac{\ell(R)^{\beta}}{|R|}\left\|\chi_{Q}\right\|_{L \mathcal{M}_{t}^{s}\left(1, w^{t}\right)}\left\|\chi_{R}\right\|_{L \mathcal{M}_{q}^{p}\left(1, w^{q}\right)^{\prime}} \lesssim \frac{\ell\left(R^{*}\right)^{\beta}}{\left|R^{*}\right|}|Q|^{\frac{1}{s}}|c(Q)|^{\beta}\left\|\chi_{R^{*}}\right\|_{L \mathcal{M}_{q}^{p}\left(1, w^{q}\right)^{\prime}} .
$$

We note that

$$
\left\|\chi_{R^{*}}\right\|_{L \mathcal{M}_{q}^{p}\left(1, w^{q}\right)^{\prime}}=\left\|w^{-1} \chi_{R^{*}}\right\|_{\left(L \mathcal{M}_{q}^{p}\right)^{\prime}} \leq\left\|w^{-1} \chi_{R^{*}}\right\|_{L^{p^{\prime}}} \lesssim \ell(R)^{\frac{n}{p^{\prime}}-\beta}
$$

where we used $\beta<\frac{n}{p^{\prime}}$ to guarantee that $\left\|w^{-1} \chi_{R^{*}}\right\|_{L^{p^{\prime}}}=\left\|\left(w_{\beta}\right)^{-1} \chi_{R^{*}}\right\|_{L^{p^{\prime}}}$ is finite. Observe also that $c(Q) \sim \ell(Q)$. As a result, since $-\frac{n}{s} \leq \beta$,

$$
\frac{\ell(R)^{\beta}}{|R|}\left\|\chi_{Q}\right\|_{L \mathcal{M}_{t}^{s}\left(1, w^{t}\right)}\left\|\chi_{R}\right\|_{L \mathcal{M}_{q}^{p}\left(1, w^{q}\right)^{\prime}} \lesssim \frac{\ell(Q)^{\beta+\frac{n}{s}}}{\ell(R)^{\beta+\frac{n}{s}}} \leq 1 .
$$

We can consider $I_{\alpha}$ by the use of Theorem 1.12.

6.5. Proof of Proposition 1.14. If we assume (1), then $\chi_{[-1,1]^{n}} \in L \mathcal{M}_{q}^{p}\left(w^{t}, w^{q}\right)$ and $\frac{\chi_{B}|\cdot|^{-n}}{\log |\cdot|} \notin L \mathcal{M}_{q}^{p}\left(w^{t}, w^{q}\right)$, where as before $B$ is a small open ball centered at 
the origin. We observe that $\chi_{[-1,1]^{n}} \in L \mathcal{M}_{q}^{p}\left(w^{t}, w^{q}\right)$ if and only if $\beta t \geq-n$ and $\frac{\chi_{B}|\cdot|^{-n}}{\log |\cdot|} \notin L \mathcal{M}_{q}^{p}\left(w^{t}, w^{q}\right)$ if and only if

$$
\beta<\frac{n s}{p^{\prime} t}
$$

If $I_{\alpha}$ is bounded from $L \mathcal{M}_{q}^{p}\left(1, w^{q}\right)$ to $L \mathcal{M}_{t}^{s}\left(1, w^{t}\right)$, then we can rule out the possibility of $\beta=-\frac{n}{t}$, since $|\cdot|^{-\frac{t \beta+n}{p}+\frac{t \beta-q \beta}{q}} \in L \mathcal{M}_{t}^{s}\left(1, w^{t}\right)$. If we assume $-\frac{n}{t}<\beta<\frac{n s}{p^{\prime} t}$, then we can argue as we did in the proof of Proposition 1.9, that $w=w_{\beta}$ satisfy

$$
\begin{aligned}
& \sup _{R \in \mathcal{Q}, R \subset 2 Q} \frac{\ell(R)^{\beta}}{|R|}\left\|\chi_{R}\right\|_{L^{t}\left(w^{t}\right)}\left\|\chi_{R}\right\|_{L^{q}\left(w^{q}\right)^{\prime}} \\
& \quad+\sup _{R \in \mathcal{Q}, R \supset Q} \frac{\ell(R)^{\beta}}{|R|}\left\|\chi_{Q}\right\|_{L \mathcal{M}_{t}^{s}\left(w^{t}, w^{t}\right)}\left\|\chi_{R}\right\|_{L \mathcal{M}_{q}^{p}\left(w^{t}, w^{q}\right)^{\prime}} \leq C
\end{aligned}
$$

for all cubes $Q \in L \mathcal{Q}$. In fact,

$$
\begin{aligned}
& \sup _{R \in \mathcal{Q}, R \subset 2 Q} \frac{\ell(R)^{\beta}}{|R|}\left\|\chi_{R}\right\|_{L^{t}\left(w^{t}\right)}\left\|\chi_{R}\right\|_{L^{q}\left(w^{q}\right)^{\prime}} \\
& \lesssim \sup _{R \in \mathcal{Q}, R \subset 2 Q} \frac{\ell(R)^{\beta} w\left(c_{R}\right)}{|R|}\left\|\chi_{R}\right\|_{L^{t}} w\left(c_{R}\right)^{-1}\left\|\chi_{R}\right\|_{\left(L^{q}\right)^{\prime}}=1 .
\end{aligned}
$$

We move on to the proof of

$$
\sup _{R \in \mathcal{Q}, R \supset Q} \frac{\ell(R)^{\beta}}{|R|}\left\|\chi_{Q}\right\|_{L \mathcal{M}_{t}^{s}\left(w^{t}, w^{t}\right)}\left\|\chi_{R}\right\|_{L \mathcal{M}_{q}^{p}\left(w^{t}, w^{q}\right)^{\prime}} \lesssim 1 .
$$

By expanding $R$ suitably, we may assume that $R$ is centered at the origin. Let

$$
\lambda=\beta\left(1+\frac{t}{p}-\frac{t}{q}\right)+\frac{n}{p}-\frac{n}{q}+\varepsilon=\beta \frac{t}{s}+\frac{n}{p}-\frac{n}{q}+\varepsilon .
$$

Here $\varepsilon>0$ is chosen small enough to have $\lambda<\frac{n}{q^{\prime}}$. This is possible because

$$
\frac{n}{q^{\prime}}-\lambda+\varepsilon=\frac{n}{p^{\prime}}-\beta\left(1+\frac{t}{p}-\frac{t}{q}\right)>\left(\frac{n s}{p^{\prime} t}-\beta\right)\left(1+\frac{t}{p}-\frac{t}{q}\right)>0 .
$$

Meanwhile,

$$
\left\|\chi_{Q}\right\|_{L \mathcal{M}_{t}^{s}\left(w^{t}, w^{t}\right)} \sim w^{t}(Q)^{\frac{1}{s}}=\ell(Q)^{\frac{n+\beta t}{s}}
$$

and for a non-negative measurable function $f$

$$
\int_{R} f(x) \mathrm{d} x \leq\left(\int_{R} f(x)^{q}|x|^{q \lambda} \mathrm{d} x\right)^{\frac{1}{q}}\left(\int_{R}|x|^{-q^{\prime} \lambda} \mathrm{d} x\right)^{\frac{1}{q^{\prime}}} \lesssim \ell(R)^{\frac{n}{q^{\prime}}-\lambda}\left(\int_{R} f(x)^{q}|x|^{q \lambda} \mathrm{d} x\right)^{\frac{1}{q}} .
$$

We borrow the idea of [7] again. We decompose

$$
\left(\int_{R} f(x)^{q}|x|^{q \lambda} \mathrm{d} x\right)^{\frac{1}{q}} \leq \sum_{j=1}^{\infty}\left(\int_{2^{1-j} R \backslash 2^{-j} R} f(x)^{q}|x|^{q \lambda} \mathrm{d} x\right)^{\frac{1}{q}}
$$


as usual. We note that

$$
\begin{aligned}
& \left(\int_{R} f(x)^{q}|x|^{q \lambda} \mathrm{d} x\right)^{\frac{1}{q}} \\
& \lesssim \sum_{j=1}^{\infty}\left(\left(2^{-j} \ell(R)\right)^{n+t \beta}\right)^{\frac{1}{q}-\frac{1}{p}} w^{t}\left(2^{-j} R\right)^{\frac{1}{p}-\frac{1}{q}}\left(\int_{2^{1-j} R \backslash 2^{-j} R} f(x)^{q}|x|^{q \lambda} \mathrm{d} x\right)^{\frac{1}{q}} \\
& \lesssim \sum_{j=1}^{\infty}\left(2^{-j} \ell(R)\right)^{\varepsilon} w^{t}\left(2^{-j} R\right)^{\frac{1}{p}-\frac{1}{q}}\left(\int_{2^{1-j} R \backslash 2^{-j} R} f(x)^{q}|x|^{q \lambda+(n+t \beta)\left(1-\frac{q}{p}\right)-q \varepsilon} \mathrm{d} x\right)^{\frac{1}{q}} \\
& =\sum_{j=1}^{\infty}\left(2^{-j} \ell(R)\right)^{\varepsilon} w^{t}\left(2^{-j} R\right)^{\frac{1}{p}-\frac{1}{q}}\left(\int_{2^{1-j} R \backslash 2^{-j} R} f(x)^{q}|x|^{q \beta} \mathrm{d} x\right)^{\frac{1}{q}} \lesssim \ell(R)^{\varepsilon}\|f\|_{\mathcal{M}_{q}^{p}\left(w^{t}, w^{q}\right)} .
\end{aligned}
$$

Thus,

$$
\int_{R} f(x) \mathrm{d} x \lesssim \ell(R)^{\frac{n}{q^{\prime}}-\lambda+\varepsilon}\|f\|_{\mathcal{M}_{q}^{p}\left(w^{t}, w^{q}\right)}
$$

We note that

$$
\beta-n+\frac{n}{q^{\prime}}-\lambda+\varepsilon=-\frac{n}{s}-\beta \frac{t}{s}
$$

Consequently,

$$
\sup _{R \in \mathcal{Q}, R \supset Q} \frac{\ell(R)^{\beta}}{|R|}\left\|\chi_{Q}\right\|_{L \mathcal{M}_{t}^{s}\left(w^{t}, w^{t}\right)}\left\|\chi_{R}\right\|_{L \mathcal{M}_{q}^{p}\left(w^{t}, w^{q}\right)^{\prime}} \lesssim \sup _{R \in \mathcal{Q}, R \supset Q} \ell(Q)^{\frac{n}{s}+\beta \frac{t}{s}} \ell(R)^{-\frac{n}{s}-\beta \frac{t}{s}}=1 .
$$

Thus, $I_{\beta}$ is bounded from $L \mathcal{M}_{q}^{p}\left(w^{t}, w^{q}\right)$ to $L \mathcal{M}_{t}^{s}\left(w^{t}, w^{t}\right)$ thanks to Theorem 1.12 . The proof for $M_{\alpha}$ is similar.

6.6. Proof of Proposition 1.18. The case of $M_{\alpha}$ is omitted because this is similar to the case of $I_{\alpha}$. If $I_{\alpha}$ is bounded from $\mathcal{M}_{q}^{p}\left(w^{t}, w^{q}\right)$ to $\mathcal{M}_{t}^{s}\left(w^{t}, w^{t}\right)$, then we have $-\frac{n}{t}<\beta<\frac{n s}{p^{\prime} t}$ as before. Conversely assume $-\frac{n}{t}<\beta<\frac{n s}{p^{\prime} t}$. Let $f \in L_{\mathrm{c}}^{\infty}$. If $\beta \leq 0$, then we can argue as Komori and Shirai did in [37]. Let us assume $\beta>0$. We distinguish four cases as before to show that

$$
w^{t}(Q)^{\frac{1}{s}-\frac{1}{t}}\left(\int_{Q}\left|I_{\alpha} f(x)\right|^{t}|x|^{\beta t} \mathrm{~d} x\right)^{\frac{1}{t}} \lesssim\|f\|_{\mathcal{M}_{q}^{p}\left(w^{t}, w^{q}\right)} .
$$

(1) If $Q$ is a cube centered at 0 and if $f$ is supported on $3 Q$, then we have

$$
\begin{aligned}
& w^{t}(Q)^{\frac{1}{s}-\frac{1}{t}}\left(\int_{Q}\left|I_{\alpha} f(x)\right|^{t}|x|^{\beta t} \mathrm{~d} x\right)^{\frac{1}{t}} \\
& \lesssim w^{t}(Q)^{\frac{1}{p}-\frac{1}{q}} \ell(Q)^{\beta-\tau}\left(\int_{Q}\left|I_{\alpha} f(x)\right|^{t}|x|^{\tau t} \mathrm{~d} x\right)^{\frac{1}{t}} .
\end{aligned}
$$

Here $\tau$ satisfies $\tau \in\left(\frac{t}{s} \beta, \beta\right)$. Since $f$ is supported on $3 Q$,

$$
\begin{aligned}
& w^{t}(Q)^{\frac{1}{s}-\frac{1}{t}} \ell(Q)^{\beta-\tau}\left(\int_{Q}\left|I_{\alpha} f(x)\right|^{t}|x|^{\tau t} \mathrm{~d} x\right)^{\frac{1}{t}} \\
& \lesssim w^{t}(Q)^{\frac{1}{s}-\frac{1}{t}} \ell(Q)^{\beta-\tau}\left(\int_{3 Q}|f(x)|^{q}|x|^{\tau q} \mathrm{~d} x\right)^{\frac{1}{q}} .
\end{aligned}
$$


We borrow the idea of [7]. From the definition of the norm,

$$
\begin{aligned}
& \left(\int_{3 Q}|f(x)|^{q}|x|^{\tau q} \mathrm{~d} x\right)^{\frac{1}{q}} \\
& \leq \sum_{j=0}^{\infty}\left(\int_{3 \cdot 2^{-j} Q \backslash 3 \cdot 2^{-j-1} Q}|f(x)|^{q}|x|^{\tau q} \mathrm{~d} x\right)^{\frac{1}{q}} \\
& \lesssim \sum_{j=0}^{\infty}\left(2^{-j} \ell(Q)\right)^{\tau-\beta}\left(\int_{3 \cdot 2^{-j} Q \backslash 3 \cdot 2^{-j-1} Q}|f(x)|^{q}|x|^{\beta q} \mathrm{~d} x\right)^{\frac{1}{q}} \\
& \lesssim \sum_{j=0}^{\infty}\left(2^{-j} \ell(Q)\right)^{\tau-\beta} w^{t}\left(2^{-j} Q\right)^{\frac{1}{q}-\frac{1}{p}}\|f\|_{\mathcal{M}_{q}^{p}\left(w^{t}, w^{q}\right)} .
\end{aligned}
$$

We observe that

$\tau-\beta+(n+t \beta)\left(\frac{1}{q}-\frac{1}{p}\right)=\tau+n\left(\frac{1}{q}-\frac{1}{p}\right)-\frac{t}{s} \beta \geq n\left(\frac{1}{q}-\frac{1}{p}\right)>0$,

where we used $\tau>\frac{t}{s} \beta$ for the penultimate inequality. Thus, the series is summable to have $(6.5)$.

(2) If $Q$ is a cube centered at 0 and if $f$ is supported outside $3 Q$, then we have

$$
w^{t}(Q)^{\frac{1}{s}-\frac{1}{t}}\left(\int_{Q}\left|I_{\alpha} f(x)\right|^{t}|x|^{\beta t} \mathrm{~d} x\right)^{\frac{1}{t}} \lesssim w^{t}(Q)^{\frac{1}{s}} \int_{\mathbf{R}^{n} \backslash 3 Q} \frac{|f(y)|}{|y|^{n-\alpha}} \mathrm{d} y
$$

from the expression of $I_{\alpha} f(x)$. We note that

$$
\begin{aligned}
& \int_{\mathbf{R}^{n} \backslash 3 Q} \frac{|f(y)|}{|y|^{n-\alpha}} \mathrm{d} y \\
& \lesssim \sum_{j=1}^{\infty} \frac{1}{\ell\left(2^{j} Q\right)^{n-\alpha}} \int_{2^{j} Q \backslash 2^{j-1} Q}|f(y)| \mathrm{d} y \\
& \lesssim \sum_{j=1}^{\infty} \ell\left(2^{j} Q\right)^{\alpha}\left(\frac{1}{\ell\left(2^{j} Q\right)^{n}} \int_{2^{j} Q \backslash 2^{j-1} Q}|f(y)|^{q} \mathrm{~d} y\right)^{\frac{1}{q}} \\
& \lesssim \sum_{j=1}^{\infty} \ell\left(2^{j} Q\right)^{\alpha-\beta+\frac{t}{q} \beta}\left(\frac{1}{w^{t}\left(2^{j} Q\right)} \int_{2^{j} Q \backslash 2^{j-1} Q}|f(y)|^{q}|y|^{q \beta} \mathrm{d} y\right)^{\frac{1}{q}} .
\end{aligned}
$$

Since

$$
\frac{1}{q}=\frac{1}{t}+\frac{\alpha}{n}, \quad \frac{1}{p}=\frac{1}{s}+\frac{\alpha}{n}
$$

we have

$$
\frac{\ell\left(2^{j} Q\right)^{\alpha-\beta+\frac{t}{q} \beta}}{w^{t}\left(2^{j} Q\right)^{\frac{1}{p}}}=\frac{\ell\left(2^{j} Q\right)^{\alpha+\frac{\alpha \beta t}{n}}}{w^{t}\left(2^{j} Q\right)^{\frac{1}{p}}}=\frac{\ell\left(2^{j} Q\right)^{\alpha+\frac{\alpha \beta t}{n}}}{w^{t}\left(2^{j} Q\right)^{\frac{\alpha}{n}} w^{t}\left(2^{j} Q\right)^{\frac{1}{s}}} \sim w^{t}\left(2^{j} Q\right)^{-\frac{1}{s}} .
$$

Hence we have

$$
\int_{\mathbf{R}^{n} \backslash 3 Q} \frac{|f(y)|}{|y|^{n-\alpha}} \mathrm{d} y \lesssim w^{t}(Q)^{-\frac{1}{s}}\|f\|_{\mathcal{M}_{q}^{p}\left(w^{t}, w^{q}\right)} .
$$

Putting these estimates all together, we obtain (6.5). 
(3) If $Q$ is a cube such that $0 \notin 32 Q$ and if $f$ is supported on $3 Q$, then by the classical Hardy-Littlewood-Sobolev theorem

$$
\begin{aligned}
w^{t}(Q)^{\frac{1}{s}-\frac{1}{t}}\left(\int_{Q}\left|I_{\alpha} f(x)\right|^{t}|x|^{\beta t} \mathrm{~d} x\right)^{\frac{1}{t}} & \lesssim w^{t}(Q)^{\frac{1}{s}-\frac{1}{t}}|c(Q)|^{\beta}\left(\int_{Q}\left|I_{\alpha} f(x)\right|^{t} \mathrm{~d} x\right)^{\frac{1}{t}} \\
& \lesssim w^{t}(Q)^{\frac{1}{s}-\frac{1}{t}}|c(Q)|^{\beta}\left(\int_{3 Q}|f(x)|^{q} \mathrm{~d} x\right)^{\frac{1}{q}} \\
& \lesssim w^{t}(3 Q)^{\frac{1}{s}-\frac{1}{t}}\left(\int_{3 Q}|f(x)|^{q}|x|^{\beta q} \mathrm{~d} x\right)^{\frac{1}{q}} \\
& \lesssim\|f\|_{\mathcal{M}_{q}^{p}\left(w^{t}, w^{q}\right)} .
\end{aligned}
$$

(4) If $Q$ is a cube such that $0 \notin 32 Q$ and if $f$ is supported outside $3 Q$, then

$$
w^{t}(Q)^{\frac{1}{s}-\frac{1}{t}}\left(\int_{Q}\left|I_{\alpha} f(x)\right|^{t}|x|^{\beta t} \mathrm{~d} x\right)^{\frac{1}{t}} \lesssim w^{t}(Q)^{\frac{1}{s}} \int_{\mathbf{R}^{n} \backslash 3 Q} \frac{|f(y)|}{|y-c(Q)|^{n-\alpha}} \mathrm{d} y
$$

from the integral expression of $f$. Consequently

$$
\begin{aligned}
& w^{t}(Q)^{\frac{1}{s}-\frac{1}{t}}\left(\int_{Q}\left|I_{\alpha} f(x)\right|^{t}|x|^{\beta t} \mathrm{~d} x\right)^{\frac{1}{t}} \\
& \lesssim w^{t}(Q)^{\frac{1}{s}} \sum_{j=1}^{\infty} \frac{1}{\ell\left(2^{j} Q\right)^{n-\alpha}} \int_{2^{j+1} Q \backslash 2^{j} Q}|f(y)| \mathrm{d} y .
\end{aligned}
$$

Let $j_{0} \geq 6$ be the smallest integer such that $0 \in 2^{j_{0}-1} Q$. Then since $|y| \sim$ $\ell\left(2^{j_{0}} Q\right)$ for all $y \in 2^{j_{0}-3} Q$, we have

$$
\begin{aligned}
& \sum_{j=1}^{j_{0}-4} \frac{1}{\ell\left(2^{j} Q\right)^{n-\alpha}} \int_{2^{j+1} Q \backslash 2^{j} Q}|f(y)| \mathrm{d} y \\
& \lesssim \sum_{j=1}^{j_{0}-4} \ell\left(2^{j} Q\right)^{\alpha}\left(\frac{1}{\ell\left(2^{j} Q\right)^{n}} \int_{2^{j+1} Q \backslash 2^{j} Q}|f(y)|^{q} \mathrm{~d} y\right)^{\frac{1}{q}} \\
& \lesssim \sum_{j=1}^{j_{0}-4} \ell\left(2^{j} Q\right)^{\alpha} \ell\left(2^{j_{0}} Q\right)^{-\beta}\left(\frac{1}{\ell\left(2^{j} Q\right)^{n}} \int_{2^{j+1} Q \backslash 2^{j} Q}|f(y)|^{q}|y|^{q \beta} \mathrm{d} y\right)^{\frac{1}{q}} \\
& \lesssim \sum_{j=1}^{j_{0}-4} \ell\left(2^{j} Q\right)^{\alpha} \ell\left(2^{j_{0}} Q\right)^{\frac{\beta t}{q}-\beta} w^{t}\left(2^{j} Q\right)^{-\frac{1}{p}}\|f\|_{\mathcal{M}_{q}^{p}\left(w^{t}, w^{q}\right)} .
\end{aligned}
$$

We note $\ell\left(2^{j} Q\right)^{\alpha} w^{t}\left(2^{j} Q\right)^{-\frac{1}{p}} \sim \ell\left(2^{j} Q\right)^{-\frac{n}{s}}|c(Q)|^{-\frac{\beta t}{p}}$, since

$$
\alpha-\frac{n}{p}=-\frac{n}{s} .
$$


As a result,

$$
\begin{aligned}
& \sum_{j=1}^{j_{0}-4} \frac{1}{\ell\left(2^{j} Q\right)^{n-\alpha}} \int_{2^{j+1} Q \backslash 2^{j} Q}|f(y)| \mathrm{d} y \\
& \lesssim \ell\left(2^{j_{0}} Q\right)^{-\beta+\frac{\beta t}{q}} \ell(Q)^{-\frac{n}{s}}|c(Q)|^{-\frac{\beta t}{p}}\|f\|_{\mathcal{M}_{q}^{p}\left(w^{t}, w^{q}\right)} \\
& \lesssim \ell\left(2^{j_{0}} Q\right)^{-\beta+\frac{\beta t}{q}-\frac{\beta t}{p}} \ell(Q)^{-\frac{n}{s}}\|f\|_{\mathcal{M}_{q}^{p}\left(w^{t}, w^{q}\right)} \\
& =\ell\left(2^{j_{0}} Q\right)^{-\frac{\beta t}{s}} \ell(Q)^{-\frac{n}{s}}\|f\|_{\mathcal{M}_{q}^{p}\left(w^{t}, w^{q}\right)} \\
& \sim w^{t}\left(2^{j_{0}} Q\right)^{-\frac{1}{s}}\|f\|_{\mathcal{M}_{q}^{p}\left(w^{t}, w^{q}\right)} .
\end{aligned}
$$

Consequently,

$$
w^{t}(Q)^{\frac{1}{s}} \sum_{j=1}^{j_{0}-4} \frac{1}{\ell\left(2^{j} Q\right)^{n-\alpha}} \int_{2^{j+1} Q \backslash 2^{j} Q}|f(y)| \mathrm{d} y \lesssim\|f\|_{\mathcal{M}_{q}^{p}\left(w^{t}, w^{q}\right)} .
$$

We use the idea of [7]. As we did in (6.4), using $\beta<\frac{n s}{p^{\prime} t}$, we have

$$
w^{t}(Q)^{\frac{1}{s}} \sum_{j=j_{0}-3}^{\infty} \frac{1}{\ell\left(2^{j} Q\right)^{n-\alpha}} \int_{2^{j+1} Q \backslash 2^{j} Q}|f(y)| \mathrm{d} y \lesssim\|f\|_{\mathcal{M}_{q}^{p}\left(w^{t}, w^{q}\right)} .
$$

Thus, putting these observations together, we obtain

$$
w^{t}(Q)^{\frac{1}{s}} \sum_{j=1}^{\infty} \frac{1}{\ell\left(2^{j} Q\right)^{n-\alpha}} \int_{2^{j+1} Q \backslash 2^{j} Q}|f(y)| \mathrm{d} y \lesssim\|f\|_{\mathcal{M}_{q}^{p}\left(w^{t}, w^{q}\right)} .
$$

All together then, we conclude that $I_{\alpha}$ is bounded from $\mathcal{M}_{q}^{p}\left(w^{t}, w^{q}\right)$ to $\mathcal{M}_{t}^{s}\left(w^{t}, w^{t}\right)$.

Acknowledgement. Shohei Nakamura was partially supported by Grand-in-Aid for Young Scientist A. 16H05995. Yoshihiro Sawano was partially supported by Grand-in-Aid for Scientific Research (C), No. 16K05209 and 19K03546 for Japan Society for the Promotion of Science and by People's Friendship University of Russia. The authors are thankful to Professor Javier Duoandikoetxea for his illuminating discussion of the power weighted Morrey spaces. Especially the authors are motivated by the paper [7].

\section{References}

[1] Adams, D. R.: A note on Riesz potentials. - Duke Math. J. 42, 1975, 765-778.

[2] BADAlov, X.: Maximal and singular integral operators and their commutators in the vanishing generalized weighted Morrey spaces with variable exponent. - Trans. Natl. Acad. Sci. Azerb. Ser. Phys.-Tech. Math. Sci. 38:1, 2018, Mathematics, 30-42.

[3] Burenkov, V.I., and E. D. Nursultanov: Description of interpolation spaces for local Morrey-type spaces. - Tr. Mat. Inst. Steklova 269, 2010, Teoriya Funktsii i Differentsialnye Uravneniya, 52-62 (in Russian); transl. in Proc. Steklov Inst. Math. 269:1, 2010, 46-56.

[4] Chen, X.: Boundedness criteria for commutators of some sublinear operators in weighted Morrey spaces. - J. Nonlinear Sci. Appl. 11:1, 2018, 26-48.

[5] Deringoz, F.: Parametric Marcinkiewicz integral operator and its higher order commutators on generalized weighted Morrey spaces. - Trans. Natl. Acad. Sci. Azerb. Ser. Phys.-Tech. Math. Sci. 37:4, 2017, Mathematics, 24-32.

[6] Duonndikoetxea, J.: Fourier analysis. - Grad. Stud. Math. 29, Amer. Math. Soc., Providence, RI, 2001. 
[7] Duonndikoetxea, J., and M. Rosenthal: Boundedness of operators on certain weighted Morrey spaces beyond Muckenhoupt range. - Submitted.

[8] Eroglu, A., M. N. Omarova, and M. A. Shemsiyye: Elliptic equations with measurable coefficients in generalized weighted Morrey spaces. - Proc. Inst. Math. Mech. Natl. Acad. Sci. Azerb. 43:2, 2017, 197-213.

[9] GAO, G. L., and X. M. Wu: Estimates of intrinsic square functions on generalized weighted Morrey spaces. - J. Funct. Spaces 2014, Art. ID 168381, 1-8.

[10] Gogatishvili, A., R. Ch. Mustafayev, and T. Ünver: Embedding relations between weighted complementary local Morrey-type spaces and weighted local Morrey-type spaces. Eurasian Math. J. 8:1, 2017, 34-49.

[11] Gong, R.: The area integral associated to self-adjoint operators on weighted Morrey spaces. - J. Math. Sci. Adv. Appl. 16:1-2, 2012, 47-59.

[12] Guliyev, V.S.: Generalized weighted Morrey spaces and higher order commutators of sublinear operators. - Eurasian Math. J. 3:3, 2012, 33-61.

[13] Guliyev, V.S., J. J. Hasanov, and X. A. Badalov: Maximal and singular integral operators and their commutators on generalized weighted Morrey spaces with variable exponent. Math. Inequal. Appl. 21:1, 2018, 41-61.

[14] Guliyev, V.S., and V. H. Hamzayev: Rough singular integral operators and its commutators on generalized weighted Morrey spaces. - Math. Inequal. Appl. 19:3, 2016, 863-881.

[15] Guliyev, V.S., T. Karaman, R. C. Mustafayev, and A. Serbetci: Commutators of sublinear operators generated by Calderon-Zygmund operator on generalized weighted Morrey spaces. - Czechoslovak Math. J. 64:2 (139), 2014, 365-386.

[16] Guliyev, V.S., S. Muradova, M. N. Omarova, and L. Softova: Gradient estimates for parabolic equations in generalized weighted Morrey spaces. - Acta Math. Sin. (Engl. Ser.) 32:8, 2016, 911-924.

[17] Guliyev, G. S., and M. N. Omarova: Higher order commutators of vector-valued intrinsic square functions on vector-valued generalized weighted Morrey spaces. - Azerb. J. Math. 4:2, $2014,64-85$.

[18] GuliYev, V.S., and M. N. Omarova: Multilinear singular and fractional integral operators on generalized weighted Morrey spaces. - Azerb. J. Math. 5:1, 2015, 104-132.

[19] GuliYev, V.S., and M. N. Omarova: Parabolic oblique derivative problem with discontinuous coefficients in generalized weighted Morrey spaces. - Open Math. 14, 2016, 49-61.

[20] Guliyev, G. S., M. N. Omarova, and Y. Sawano: Boundedness of intrinsic square functions and their commutators on generalized weighted Orlicz-Morrey spaces. - Banach J. Math. Anal. $9: 2,2015,44-62$.

[21] Hamzayev, V.H.: Sublinear operators with rough kernel generated by Calderón-Zygmund operators and their commutators on generalized weighted Morrey spaces. - Trans. Natl. Acad. Sci. Azerb. Ser. Phys.-Tech. Math. Sci. 38:1, 2018, Mathematics, 79-94.

[22] Haroske, D. D., and L. Skrzypczak: Embeddings of weighted Morrey spaces. - Math. Nachr. 290:7, 2017, 1066-1086.

[23] HE, Y.X.: Toeplitz operator related to singular integral with non-smooth kernel on weighted Morrey space. - Anal. Theory Appl. 33:3, 2017, 240-252.

[24] Ho, K. P.: Vector-valued maximal inequalities on weighted Orlicz-Morrey spaces, Tokyo J. Math. 36 (2013), no. 2, 499-512.

[25] Hu, Y., Z. LI, and Y. WANG: Multilinear singular integral operators on generalized weighted Morrey spaces. - J. Funct. Spaces 2014, Art. ID 325924, 1-12.

[26] Hu, X., and J. ZHOU: Pseudodifferential operators with smooth symbols and their commutators on weighted Morrey spaces. - J. Pseudo-Differ. Oper. Appl. 9:2, 2018, 215-227. 
[27] Hytönen, T.P.: The $A_{2}$ theorem: remarks and complements. - In: Harmonic analysis and partial differential equations 612, Contemp. Math. Amer. Math. Soc., Providence, RI, 2014, 91-106.

[28] IIDA, T.: The boundedness of the Hardy-Littlewood maximal operator and multilinear maximal operator in weighted Morrey type spaces. - J. Funct. Spaces 2014, Art. ID 648251, 1-8.

[29] IIDA, T.: Weighted inequalities on Morrey spaces for linear and multilinear fractional integrals with homogeneous kernels. - Taiwanese Math. J. 18:1, 2014, 147-185.

[30] IIDA, T.: Various inequalities related to the Adams inequality on weighted Morrey spaces. Math. Inequal. Appl. 20:3, 2017, 601-650.

[31] Iida, T., Y. Komori-Furuya, and E. Sato: New multiple weights and the Adams inequality on weighted Morrey spaces. - Sci. Math. Jpn. 74:2-3, 2011, 145-157.

[32] Iida, T., Y. Komori-FuruYA, and E. Sato: The Adams inequality on weighted Morrey spaces. - Tokyo J. Math. 34:2, 2011, 535-545.

[33] Iida, T., and S. Nakamura: A note on the Feerman-Stein inequality on Morrey spaces. Rev. Mat. Complut. 30:3, 2017, 525-545.

[34] Iida, T., E. Sato, Y. Sawano, and H. Tanaka: Weighted norm inequalities for multilinear fractional operators on Morrey spaces. - Studia Math. 205, 2011, 139-170.

[35] Karaman, T., V.S. Guliyev, and A. Serbetci: Boundedness of sublinear operators generated by Calderon-Zygmund operators on generalized weighted Morrey spaces. - An. Stiint. Univ. Al. I. Cuza Iasi. Mat. (N.S.) 60:1, 2014, 227-244.

[36] Komori-Furuya, Y., and E. Sato: Weighted estimates for fractional integral operators on central Morrey spaces. - Math. Nachr. 2017, 290, 2901-2908.

[37] Komori, Y., and S. Shirai: Weighted Morrey spaces and a singular integral operator. - Math. Nachr. 282:2, 2009, 219-231.

[38] Lerner, A. K.: A simple proof of the $A_{2}$ conjecture. - Int. Math. Res. Not. 2013:14, 2013, 3159-3170.

[39] LiU, L.Z.: Sharp and weighted boundedness for multilinear operators of pseudo-differential operators on Morrey space. - Izv. Nats. Akad. Nauk Armenii Mat. 45:3, 2010, 57-72; transl. in J. Contemp. Math. Anal. 45:3, 2010, 136-150.

[40] LiU, Y., and L. J. WANG: Boundedness for Riesz transform associated with Schrodinger operators and its commutator on weighted Morrey spaces related to certain nonnegative potentials. - J. Inequal. Appl. 2014:194, 2014, 1-16.

[41] Lukkassen, D., A. Meidell, L. E. Persson, and N. Samko: Hardy and singular operators in weighted generalized Morrey spaces with applications to singular integral equations. - Math. Methods Appl. Sci. 35:11, 2012, 1300-1311.

[42] Muckenhoupt, B.: Weighted norm inequalities for the Hardy maximal function. - Trans. Amer. Math. Soc. 165, 1972, 207-226.

[43] Mustafayev, R. Ch.: On boundedness of sublinear operators in weighted Morrey spaces. Azerb. J. Math. 2:1, 2012, 66-79.

[44] Nakamura, S.: Generalized weighted Morrey spaces and classical operators. - Math. Nachr. 289:17-18, 2016, 2235-2262.

[45] Nakamura, S., and Y. SAwano: The singular integral operator and its commutator on weighted Morrey spaces. - Collect. Math. 68:2, 2017, 145-174.

[46] Nakamura, S., Y. Sawano, and H. Tanaka: The fractional operators on weighted Morrey spaces. - J. Geom. Anal. 28:2, 2018, 1502-1524.

[47] SAmкo, N.: Fredholmness of singular integral operators in weighted Morrey spaces. - Proc. A. Razmadze Math. Inst. 148, 2008, 51-68.

[48] Samko, N.: Weighted Hardy and singular operators in Morrey spaces. - J. Math. Anal. Appl. $350: 1,2009,56-72$. 
[49] SHI, S. G.: Estimates for vector-valued commutators on weighted Morrey space and applications. - Acta Math. Sin. 29:5, 2013, 883-896.

[50] Shi, S. G., Z. Fu, and F. Zhнo: Estimates for operators on weighted Morrey spaces and their applications to nondivergence elliptic equations. - J. Inequal. Appl. 2013:390, 2013.

[51] Si, Z. G., and F. Y. ZHAO: Necessary and sufficient conditions for boundedness of commutators of the general fractional integral operators on weighted Morrey spaces. - Abstr. Appl. Anal. 2012, Art. ID 929381, 1-14.

[52] Spanne, S.: Sur l'interpolation entres les espaces $\mathcal{L}_{k}^{(p, \Phi)}$. - Ann. Scuola Norm. Sup. Pisa 20, $1966,625-648$.

[53] Tanaka, H.: Two-weight norm inequalities on Morrey spaces. - Ann. Acad. Sci. Fenn. Math. 40, 2015, 773-791.

[54] Wang, H.: Some estimates for commutators of fractional integrals associated to operators with Gaussian kernel bounds on weighted Morrey spaces. - Anal. Theory Appl. 29:1, 2013, $72-85$.

[55] WANG, Z. H., and Z. G. Si: Commutator theorems for fractional integral operators on weighted Morrey spaces. - Abstr. Appl. Anal. 2014, Art. ID 413716, 1-8.

[56] XIE, P. Z., and G.F. CAO: Toeplitz-type operators in weighted Morrey spaces. - J. Inequal. Appl. 2013:253, 2013, 1-9.

[57] YANG, D., and S. YANG: Elementary characterizations of generalized weighted MorreyCampanato spaces. - Appl. Math. J. Chinese Univ. Ser. B 25:2, 2010, 162-176.

[58] YANG, D., and S. YANG: New characterizations of weighted Morrey-Campanato spaces. Taiwanese J. Math. 15:1, 2011, 141-163.

[59] YE, X.F.: Some estimates for multilinear commutators on the weighted Morrey spaces. Math. Sci. (Springer) 6, 2012, Art. 33, 1-6.

[60] Ye, X.F., and T.F. WAng: Two-weighted norm inequality on weighted Morrey spaces. Turkish J. Math. 38:3, 2014, 426-435.

[61] YE, X.F., and X.S. ZHU: Estimates of singular integrals and multilinear commutators in weighted Morrey spaces. - J. Ineq. Appl. 2012:302, 2012.

[62] YU, Y., J. Chen, and H. LIN: Weighted Morrey spaces on non-homogeneous metric measure spaces. - J. Math. Anal. Appl. 452, 2017, 335-350.

[63] ZhANG, J., and H. Wu: Oscillation and variation inequalities for singular integrals and commutators on weighted Morrey spaces. - Front. Math. China 11:2, 2016, 423-447.

Received 6 November 2018 • Accepted 17 January 2019 